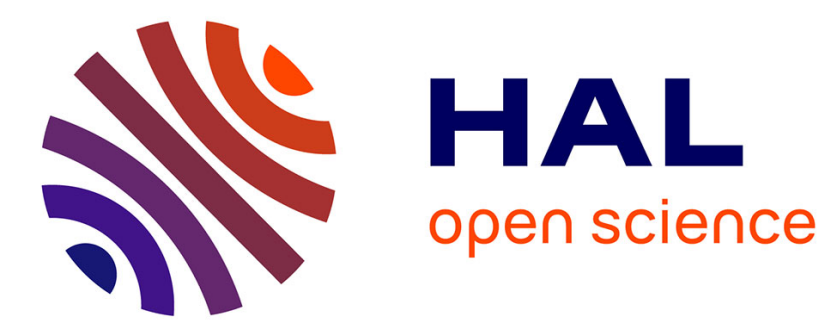

\title{
Ral GTPases promote metastasis by controlling biogenesis and organ colonization of exosomes
}

S Ghoroghi, B Mary, A Larnicol, A Klein, N Osmani, I Busnelli, F Delalande, N Paul, S Halary, F Gros, et al.

\section{- To cite this version:}

S Ghoroghi, B Mary, A Larnicol, A Klein, N Osmani, et al.. Ral GTPases promote metastasis by controlling biogenesis and organ colonization of exosomes. 2020. hal-02991406

\author{
HAL Id: hal-02991406 \\ https://hal.science/hal-02991406
}

Preprint submitted on 12 Nov 2020

HAL is a multi-disciplinary open access archive for the deposit and dissemination of scientific research documents, whether they are published or not. The documents may come from teaching and research institutions in France or abroad, or from public or private research centers.
L'archive ouverte pluridisciplinaire HAL, est destinée au dépôt et à la diffusion de documents scientifiques de niveau recherche, publiés ou non, émanant des établissements d'enseignement et de recherche français ou étrangers, des laboratoires publics ou privés. 


\title{
Ral GTPases promote metastasis by controlling biogenesis and organ colonization of exosomes
}

\author{
S Ghoroghi $\mathbf{i}^{1,2,3}$, B Mary ${ }^{1,2,3}$, A Larnicol $^{1,2,3}$, A Klein $^{1,2,3}, \mathbf{N ~ O s m a n i ~}^{1,2,3}$, I Busnelli ${ }^{1,2,3}$, F Delalande $^{4}, \mathbf{N ~ P a u l}^{1,2,3}$, S Halary $^{5}, \mathbf{F}$ \\ Gros $^{1,2,3}$, L Fouillen ${ }^{6}$, AM Haeberle ${ }^{7}$, C Royer ${ }^{8}$, C Spiegelhalter ${ }^{9}$, G André-Grégoire ${ }^{10,11}$, K Murphy $^{12,13}$, P Timpson ${ }^{12,13}, \mathbf{R}$

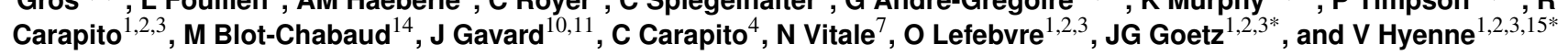 \\ ${ }^{1}$ INSERM UMR S1109, Tumor Biomechanics, Strasbourg, France \\ ${ }^{2}$ Université de Strasbourg, Strasbourg, France \\ ${ }^{3}$ Fédération de Médecine Translationnelle de Strasbourg (FMTS), Strasbourg, France \\ ${ }^{4}$ Laboratoire de Spectrométrie de Masse BioOrganique (LSMBO), IPHC UMR 7178, CNRS, Université de Strasbourg, Strasbourg, France \\ ${ }^{5}$ CNRS, UMR 7245 MCAM, Muséum National d'Histoire Naturelle de Paris, Paris, France \\ ${ }^{6}$ Université de Bordeaux, CNRS, Laboratoire de Biogenèse Membranaire, UMR 5200, 33140, Villenave d'Ornon, France \\ ${ }^{7}$ Centre National de la Recherche Scientifique, Université de Strasbourg, Institut des Neurosciences Cellulaires et Intégratives, F-67000 Strasbourg, France \\ ${ }^{8}$ Plateforme Imagerie In Vitro, CNRS UPS 3156, Strasbourg, France \\ ${ }^{9}$ IGBMC Imaging Center CNRS (UMR7104)/ INSERM (U1258)/ Université de Strasbourg, Illkirch, France \\ ${ }^{10}$ Team SOAP, CRCINA, INSERM, CNRS, Université de Nantes, Université d'Angers, Nantes, France \\ ${ }^{11}$ Integrated Center for Oncology, ICO, St-Herblain, France \\ ${ }^{12}$ The Kinghorn Cancer Centre, Garvan Institute of Medical Research, Sydney, New South Wales 2010, Australia \\ ${ }^{13}$ Vincent's Clinical School, Faculty of Medicine, University of New South Wales, Sydney, New South Wales 2010, Australia \\ ${ }^{14}$ C2VN, INSERM 1263, Inrae 1260, Aix-Marseille Université, 27 Bd J. Moulin, 13005 Marseille, France \\ ${ }^{15}$ CNRS SNC5055, Strasbourg, France \\ *Equal contribution
}

\begin{abstract}
Cancer extracellular vesicles (EVs) mainly exert pro-tumoral functions by changing the phenotypes of stromal cells to the benefit of tumor growth and metastasis. They shuttle to distant organs and fertilize pre-metastatic niches facilitating subsequent seeding by circulating tumor cells. The levels of tumor secreted EVs correlate with tumor aggressiveness, however, the link between EV secretion mechanisms and their capacity to form premetastatic niches remains obscure. Here, we show that GTPases of the Ral family control, through the phospholipase D1, multivesicular bodies homeostasis and thereby tune the biogenesis and secretion of pro-metastatic EVs. RalA and RalB promote lung metastasis in a syngeneic mouse model. Importantly, EVs from RalA or RalB depleted cells have limited organotropic capacities in vivo and, as a consequence, are less efficient in promoting lung metastasis. RalA or RalB modulate the EV levels of the adhesion molecule MCAM/CD146, which mediates lung colonization. Finally, RalA and RalB, but also MCAM/CD146, are factors of poor prognosis in human breast cancer patients. Altogether, our study identifies Ral GTPases as central molecules linking the mechanisms of EVs secretion, cargo loading to their capacity to disseminate and induce pre-metastatic niches.
\end{abstract}

Exosome Ral GTPase pre-metastatic niche

Correspondence: hyenne@unistra.fr jacky.goetz@inserm.fr

\section{Introduction}

The communication between tumor cells and their neighboring stromal cells is essential to sustain tumor growth and promote invasion and metastasis(Becker et al., 2016; Follain et al., 2020). Notably, this communication allows tumors to indoctrinate their microenvironment and switch the phenotypes of various cell types, such as endothelial cells, fibroblasts or immune cells to the benefit of tumor growth, invasion, immune escape and metastasis. Such communication occurs with organs distant of the primary tumors and favors the formation of pre-metastatic niches where the modified microenvironment can help settling metastatic tumor cells(Peinado et al., 2017). Seeding of this favorable metastatic environment can be mediated by soluble molecules(Kaplan et al., 2005; Wang et al., 2017) or by extracellular vesicles (EVs) secreted by tumor cells(Costa-Silva et al., 2015; Hoshino et al., 2015; Jung et al., 2009; Peinado et al., 2012). EVs are lipid bilayered vesicles of nanometric diameters containing a complex mixture of RNA and protein cargoes, including a repertoire of surface receptors(Mathieu et al., 2019). They can be directly secreted from the plasma membrane and called microvesicles or originate from an endosomal compartment, the multi-vesicular body (MVB), and then called exosomes(van Niel et al., 2018). The levels of circulating tumor EVs tend to correlate with tumor progression(Baran et al., 2010; Galindo-Hernandez et al., 2013; Logozzi et al., 2009). Accordingly, inhibition of key components of the EV secretion machinery often correlates with decreased metastasis(Hyenne et al., 2017). For instance, Rab27a, which directs exosome secretion by controlling the docking of MVBs to the plasma membrane(Ostrowski et al., 2010), promotes breast and melanoma tumor growth and metastasis in mice(Bobrie et al., 2012; Peinado et al., 2012) and predicts poor survival in human pancreatic cancer(Wang et al., 2015). In addition to the levels of secreted tumor EVs, their content, and in particular their set of surface adhesion proteins equally orchestrates metastasis formation. For instance, the presence of tetraspanins CD151 and Tspan8 on the surface of pancreatic adenocarcinoma EVs favors metastasis in rats by enhancing their adhesive capacities and controlling their biodistribution(Yue et al., 2015). Moreover, integrin receptors exposed by tumor EVs dictate their organotropism and thereby tune/control the seeding of a premetastatic niche in specific and distant organ(Hoshino et al., 2015). Therefore, accumulating evidence show that both the levels and the content of secreted tumor EVs are instrumental in promoting metastasis. However, the molecular mechanisms coordinating these pro- 
cesses remain elusive. In particular, how the machinery governing EV secretion can impact the pro-metastatic properties of tumor EVs deserves in-depth characterization. To address this issue, we focused on the members of the Ral family, RalA and RalB (collectively referred to as RalA/B), acting downstream of RAS and promoting metastasis of different tumor types in both mice and human(Gentry et al., 2014; Yan and Theodorescu, 2018). We recently found that these versatile proteins are evolutionarily conserved regulators of exosome secretion(Hyenne et al., 2015). We originally observed that, in the nematode C. elegans, the Ral GTPase ortholog RAL-1 controls exosome secretion by acting on the biogenesis of MVBs. Importantly, we further showed that RalA/B modulate the levels of secreted EVs in models that are relevant to human breast cancer (Hyenne et al., 2015) suggesting that these GTPases could influence disease progression through EVs release. Here, we exploited 4T1 cells, an aggressive mammary tumor model that mimics human triplenegative breast cancer (Kaur et al., 2012) to further decipher how RalA/B tune EV secretion mechanisms and thereby control metastatic progression of the disease. In this study, we first provide a detailed dissection of the impact of the Ral GTPases on EV secretion levels and unravel the mechanisms by which they control the homeostasis of MVBs. RalA/B directly acts through the phospholipase D1 (PLD1), which, as we show, also promotes EVs secretion, to favor the maturation of MVBs. We further demonstrate that RalA and RalB promote lung metastasis without affecting the invasive potential of breast carcinoma. Importantly, RalA/B are crucial for the organ targeting of tumor EVs, and, as a consequence, for the seeding of pre-metastatic niches. Finally, we identify the adhesion protein CD146/MCAM as a key EV cargo controlled by RalA and RalB and demonstrate that it conveys, in part, the pro-metastatic function to EVs by controlling the lung tropism of breast cancer EVs.

\section{Results}

RalA and RalB control exosome secretion levels through the homeostasis of MVBs We have previously shown that RalA and RalB control EV secretion in aggressive 4T1 mammary tumor cells (Hyenne et al., 2015). We thus built on this relevant tumor model and decided to test the hypothesis that RalA and RalB could orchestrate pro-metastatic functions by tuning the molecular mechanisms driving the secretion levels and nature of EVs. We first confirmed our initial observations with the nanoparticle tracking analysis (NTA) of EVs released by 4T1 cells and isolated by ultracentrifugation (100.000g pellet). Stable depletion of RalA or RalB by shRNA reduces by 40per cent the amount of secreted EVs (Fig.1a), with no impact on their average size (Supplementary Figure 1a). RBC8 and BQU57, two previously described specific chemical inhibitors of Ral GTPases (Yan et al., 2014) significantly reduced EV secretion levels in mouse and human mammary tumor cell lines (4T1 and MDA-MB231 cells, respectively) as well as in human melanoma (A375) and pancreatic carcinoma (Panc1) cells (Figure 1b). Together with evidence previously obtained in
C. elegans (Hyenne et al., 2015), this demonstrates that the mechanisms by which RalA/B GTPases tune EV secretion levels are conserved throughout evolution and are notably at play in various cancer cell lines. To better understand how Ral GTPases could impact EVs secretion, we first characterized their intracellular distribution in 4T1 cells. Endogenous RalA and RalB localize mostly within CD63-positive endosomal compartments (MVBs and late endosomes), as well as at the plasma membrane (Figure 1c). Similarly, GFP-tagged RalA and RalB localize both in late endosomal compartments positive for Lysotracker and at the plasma membrane (Figure 1c). Therefore, in $4 \mathrm{~T} 1$ cells, Ral GTPases localize both at biogenesis sites of microvesicles (plasma membrane) and exosomes (MVBs). To further determine whether Ral GTPases affect MVBs as previously observed in C. elegans, we performed thorough electron microscopy (EM) analysis of endosomal compartments in 4T1 cells. In a first analysis of cells that were processed upon chemical fixation, we quantified the densities of i) MVBs and ii) endolysosomes, as well as iii) the diameter of MVBs, iv) the number and v) the diameter of intraluminal vesicles (ILVs) per MVB. Strikingly, we found RalA or RalB depletion leads to a 40per cent decrease in the number of MVB per cytoplasmic surface in $4 \mathrm{~T} 1$ cells (Figure 1d and Supplementary Figure 2a), with no impact on the density of endolysosomes (Supplementary Figure 2b). Further analysis of Lysotracker positive compartments using FACS confirmed that RalA/B depletion has no significant effect on the late endosome-lysosome pathway (Supplementary Figure 2c). Besides, EM analysis revealed no differences in ILV numbers per MVB surface (Supplementary Figure 2d), nor in MVB diameters (Supplementary Figure 2e). However, since chemical fixation is known to affect the morphology of endosomal compartments, we took our EM analysis one step forward by implementing high-pressure freezing (HPF) of cells, which better preserves the ultrastructure of endosomes (Klumperman and Raposo, 2014). A similar decrease in the number of MVBs per cytoplasmic surface in RalA and RalB knockdown cells was observed in these conditions (Supplementary Figure 2a). Upon HPF, we further observed a slight decrease in the number of ILVs per MVB surface (Supplementary Figure 2d) that could be, in part, explained by a slight increase in MVB diameters (Supplementary Figure 2e). In conclusion, depletion of either RalA or RalB significantly reduces MVB number, while the remaining MVBs are slightly bigger. Overall, thorough EM analysis of intracellular compartments using both chemical fixation and HPF clearly demonstrates that both RalA and RalB control MVB homeostasis in breast mammary tumor cells.

A RalA/B-PLD1-PA axis governs exosome biogenesis We further investigated the molecular mechanisms controlling MVB homeostasis downstream of RalA/B GTPases. We decided to focus on phospholipases D (PLDs), which catalyzes the hydrolysis of phosphatidylcholine (PC) into phosphatidic acid (PA), for three reasons: 1) PLD1 and PLD2 are two well-known targets of RalA and RalB (Jiang et al., 1995; Luo et al., 1998; Vitale et al., 2005), 2) PLD2 controls exosome secretion in breast cancer cells (Ghossoub et 
bioRxiv preprint doi: https://doi.org/10.1101/2020.07.10.196691; this version posted July 10, 2020. The copyright holder for this preprint (which was not certified by peer review) is the author/funder. All rights reserved. No reuse allowed without permission.
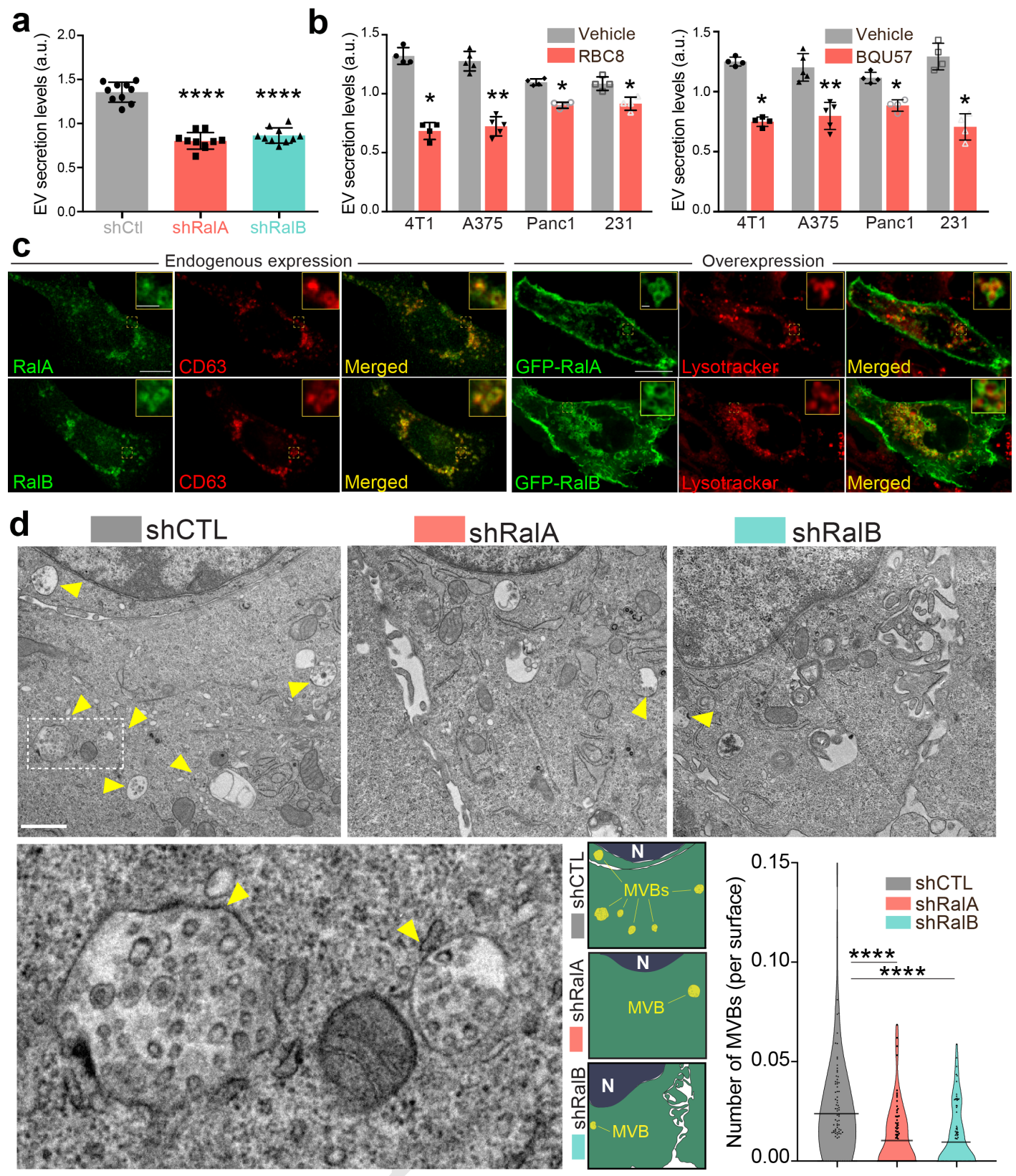

Fig. 1. RalA and RalB control exosome secretion and MVB homeostasis. a-b) Nanoparticle tracking analysis of EVs isolated by ultracentrifugation (100.000g pellet) from the supernatant of shCtl, shRalA or shRalB 4T1 cells (a) or from various cell types treated with Ral inhibitors RBC8 (b, left) or BQU57 (b, right). 231 : MDA-MB-231 cells. Each dot represents one experiment (a: 10 independent experiments; one Way Anova followed by Bonferroni's Multiple Comparison Test; b: 4 to 5 independent experiments, Mann Whitney test). c) Representative confocal images of $4 \mathrm{~T} 1$ cells showing endogenous expression of RalA, RalB and CD63 by immunofluorescence (left) and overexpression of GFP-RalA and GFP-RalB in cells incubated with Lysotracker (right). Scale bar: $10 \mu \mathrm{m}$; zoom: $2 \mu \mathrm{m}$. d) Representative electron micrographs of 4T1 shCtl, shRalA and shRalB cells, with zoom on MVBs; Scale bar: $1 \mu \mathrm{m}$; zoom: $200 \mathrm{~nm}$. Violin plots show quantification of the number of MVB per cytoplasm surface. Each dot represents one field of view; horizontal bars represent the average (76 to 88 fields of view; Kruskal-Wallis test followed by Dunn's Multiple Comparison Test).

al., 2014) and 3) PLDs impact cancer progression (Bruntz et al., 2014). We first verified that both PLD1 and PLD2 are expressed in 4T1 cells by RT-qPCR (Supplementary Figure 3a). In the absence of efficient anti-PLD antibody for immunofluorescence, we decided to assess the subcellular localization of PLD-GFP fusion proteins. PLD1 mostly localizes to endosomal compartments positive for RalA, RalB and lysotracker, whereas PLD2 mostly localizes to the plasma membrane (Figure 2a and Supplementary Figure 3b). Therefore, we tested whether PLDs could function downstream of RalA/B to control MVBs homeostasis and exosome secre- tion using two chemical inhibitors, CAY10593 for PLD1 and CAY10594 for PLD2 (Lewis et al., 2009; Scott et al., 2009). EM analysis of 4T1 cells revealed that inhibition of PLD1, but not of PLD2, induces a 40per cent decrease in the number of MVBs per cytoplasmic surface (Figure 2b). This phenotype is consistent with PLDs respective localizations and suggests that PLD1 functions in the RalA/B exosome secretion pathway. Further NTA analysis of treated cells showed that both inhibitors reduce EV secretion levels in 4T1 cells (Figure 2c), suggesting that both PLD isoforms regulate EV secretion potentially through distinct mechanisms. Importantly, 
PLD1 inhibition fully phenocopies the effect of RalA/B GTPases depletion, both on the cellular density of MVBs and on the level of EV secretion. To determine whether PLD1 acts downstream of RalA/B, we looked at its localization in the absence of RalA or RalB. Confocal analysis revealed that in 40per cent of shRalA or shRalB cells, PLD1 is uniformly cytoplasmic instead of being endosomal (Figure 2d). By contrast, RalA/B depletion had no major impact on PLD2 localization at the plasma membrane (also its trafficking might be altered) (Supplementary Figure 3c). This shows that RalA/B GTPases are required for PLD1 localization on endosomes. To further investigate if PLD activity is involved in Ral GTPases dependent EV secretion, we performed a lipidomic analysis of secreted EVs. As PLD converts PC into PA, we focused on these two lipid species. Importantly, RalA/B depletion significantly reduces the PA/PC ratio of secreted EVs (Figure 2e). In particular, the PA/PC ratio made of monoand di-unsaturated lipid species (36:1, 36:2, 38:1 and 38:2), known to be PLD product/target, respectively, showed a tendency to be decreased although not reaching statistical significance (Supplementary Figure 3d). This further implies that PLD's main product, PA, plays a crucial role in MVB homeostasis. Altogether, these results suggest that Ral GTPases control PLD1 localization on MVBs, which is required for local PA accumulation and ultimately for MVB homeostasis and exosome secretion (Figure 2f).

RalA and RalB promote metastasis non-cell autonomously Having identified RalA and RalB as important regulators of $\mathrm{EV}$ secretion in breast cancer cells, we next investigated whether such a function could impact metastasis. At first, we analyzed public databases to interrogate a potential correlation between RalA/B expression levels and metastatic progression. Using a large cohort of breast cancer patients with metastatic progression from the Cancer Genome Atlas (TCGA), we found that high expression of either RalA or RalB is significantly correlated with reduced survival (Figure 3a). Automated quantification of RalA/B expression levels by immunohistochemistry in primary tumors of breast cancer patients unraveled overexpression of both proteins in tumors from patients with metastasis (Figure 3b). These results prompted us to investigate in depth the role of RalA/B in a syngeneic mouse model of aggressive breast cancer, which is highly relevant to the human pathology. Therefore, we conducted a careful and exhaustive longitudinal analysis of metastatic progression of mammary tumors in syngeneic Balb/c mice. Briefly, 4T1 cells depleted or not for RalA or RalB were orthotopically grafted in mammary ducts, and several criteria were tracked over time. First, RalA and RalB have antagonist effects on tumor growth measured in vivo over time and ex vivo after 41 days: while RalA depletion significantly increased tumors growth, RalB depletion induced the opposite effect when compared to control tumors (Figure 3c). Neither RalA, nor RalB affected apoptosis, using caspase3 as a read-out (Supplementary Figure 4a-b). In contrast, depletion of RalA and RalB increased the growth rate of $4 \mathrm{~T} 1$ cells in vitro (Supplementary Figure 4c). A similar increase in proliferation rates was observed in vivo in the absence of RalA (Figure 3d). Therefore, while depletion of RalA favors in vivo tumor growth by enhancing 4T1 proliferation potential, it is likely that additional non-cell autonomous factors are responsible for the decreased tumor growth observed upon RalB depletion. We obtained the most striking result when carefully assessing the lung metastasis burden of these mice after 41 days. We measured the number and the surface covered by metastatic foci in serial lung sections and observed that RalA or RalB depletion in mammary tumors drastically reduced their metastatic potency (Figure $3 e)$. When compared to the tumor growth rate, the most dramatic reduction of metastasis was observed in the case of RalA depletion. These experiments show that although RalA and RalB have antagonist effects on primary tumors, they both promote metastasis. To dissect this phenotype, we tested whether RalA or RalB could impact inherent cell migration and invasion potential of $4 \mathrm{~T} 1$ cells, as it had been reported for RalB (Oxford et al., 2005; Zago et al., 2018). We performed 2D (Figure 3f) and 3D (Figure 3g) in vitro invasion assays and observed no effect of RalA or RalB expression levels on motility potential of $4 \mathrm{~T} 1$ cells. Therefore, RalA/B promote metastasis independently of cell invasion and are likely to promote metastasis of aggressive breast cancer cells non-cell autonomously by inducing pro-metastatic micro-environmental changes.

RalA and RalB dependent EVs induce endothelial permeability Since RalA and RalB promote metastasis independently of their cell-intrinsic properties, we wondered whether they could control secreted factors that are likely to induce micro-environmental alterations. In addition to EVs, tumor cells secreted soluble factors can promote metastasis by modulating the microenvironment, notably by promoting the formation of a metastatic niche (Ombrato et al., 2019). To test this possibility, we examined the impact of RalA and RalB on the soluble secretome of 4T1 cells. Depletion of RalA or RalB had no drastic effect on the soluble factors secreted by 4T1 cells (Supplementary Figure 5). However, the secretion of one protein known to promote metastasis (Ombrato et al., 2019), WISP1/CCN4, is significantly decreased in shRalA/B cells (Supplementary Figure 5). Thus, RalA and RalB are likely to enhance metastatic potency by promoting the secretion of EVs and WISP1/CCN4. Furthermore, in addition to enhancing the levels of secreted EVs, RalA/B could alter their functionality. To test this possibility, we challenged the pro-tumoral function of RalA/B EVs in an in vitro functional assay. Since tumor EVs are known to induce vascular permeability in the vicinity of tumors as well as in distant organs (Tominaga et al., 2015; Treps et al., 2016; Zhou et al., 2014), we tested the capacity of RalA/B dependent EVs to promote endothelial permeability in vitro. When added to a monolayer of endothelial cells, 4T1 EVs increased its permeability in a dose-dependent manner (Supplementary Figure 4d). We then tested the impact of EV content on vascular permeability by subjecting endothelial cells to similar amounts of EVs derived from 4T1 cells expressing or not RalA/B. Interestingly, endothelial monolayers became less permeable when 
bioRxiv preprint doi: https://doi.org/10.1101/2020.07.10.196691; this version posted July 10, 2020. The copyright holder for this preprint (which was not certified by peer review) is the author/funder. All rights reserved. No reuse allowed without permission.

a
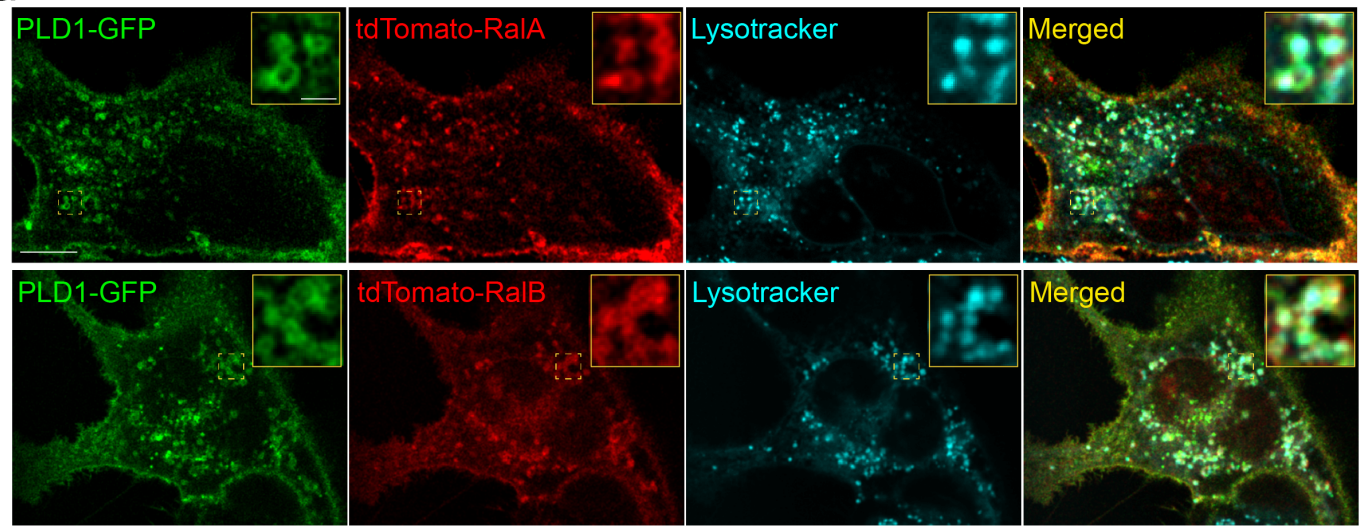

b
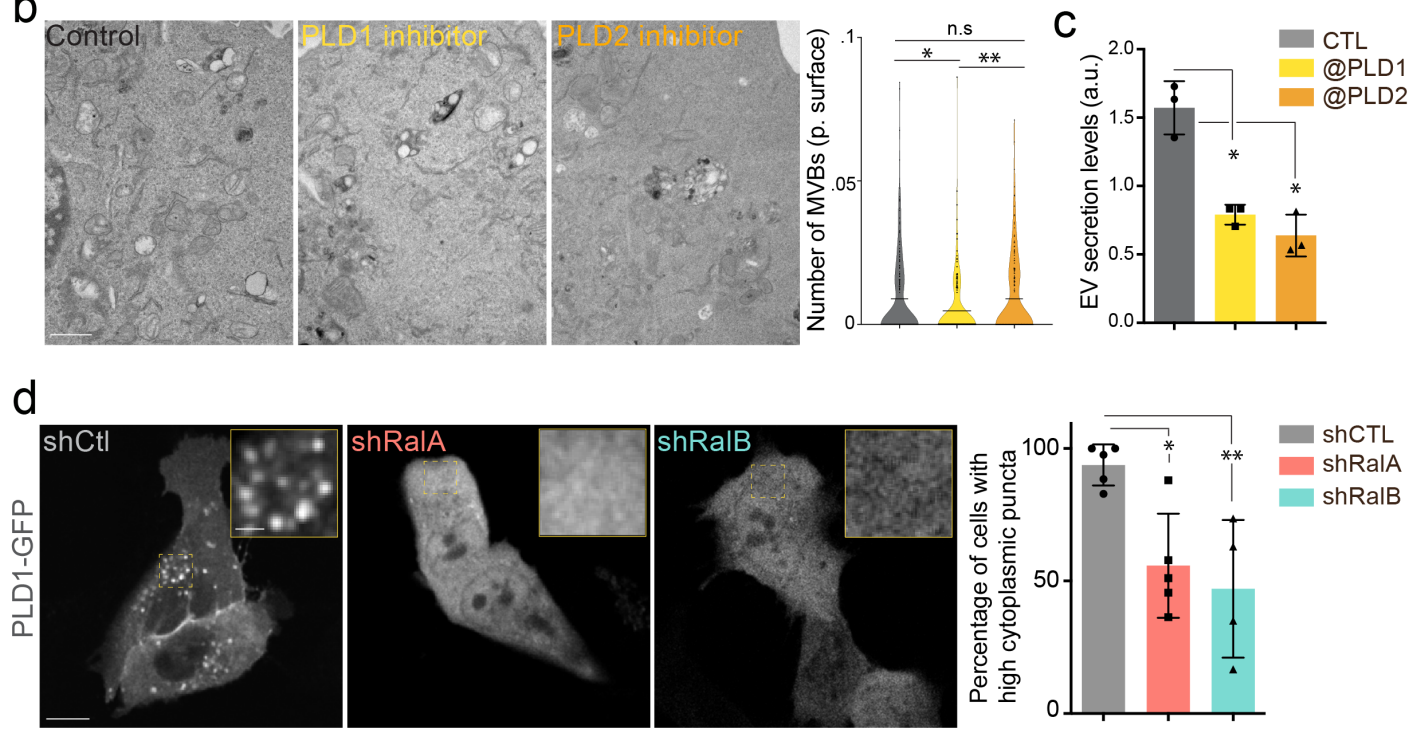

e
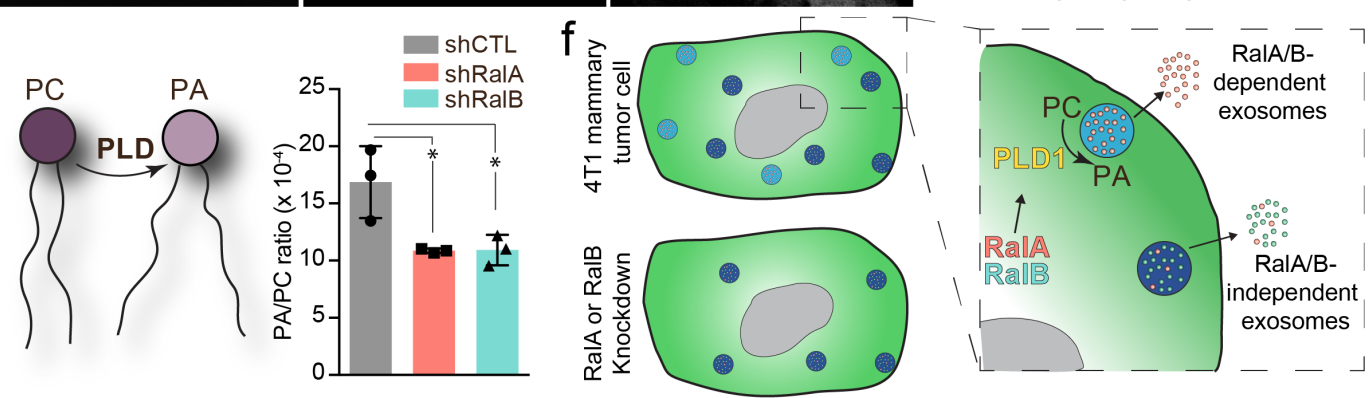

Fig. 2. The RalA/B-PLD1-PA axis governs exosome secretion. a) Representative confocal images of $4 T 1$ cells co-transfected with PLD1-GFP and tdTomato-RalA (upper panels) or tdTomato-RalB (Lower panels) and incubated with Lysotracker. Scale bar: $10 \mu \mathrm{m}$; zoom: $2 \mu \mathrm{m}$. b) Electron microscopy analysis of 4T1 cells treated with PLD1 or PLD2 inhibitor. Scale bar: $1 \mu \mathrm{m}$. Violin plots show quantification of the number of MVB per cytoplasmic surface. Each dot represents one field of view; horizontal bar represents the average (180 to 194 fields of view; Kruskal-Wallis test followed by Dunn's Multiple Comparison Test). c) Nanoparticle tracking analysis of EVs isolated by ultracentrifugation (100.000g pellet) from the supernatant of 4T1 cells treated with PLD1 (CAY10593) or PLD2 (CAY10594) inhibitor. Each dot represents one experiment (3 independent experiments; One Way Anova permutation test followed with fdr multi-comparison permutation test with fdr correction). d) Representative confocal images of shControl, shRalA and shRalB 4T1 cells transfected with PLD1-GFP. Scale bar: $10 \mu \mathrm{m}$; zoom: $2 \mu \mathrm{m}$. Graph shows the percentage of cells with high (>5) number of PLD1-GFP cytoplasmic puncta. (Each dot represents one experiment. 5 independent experiments; Number of cells analyzed: shCtl (136), shRalA (170), shRalB (244); Kruskal-Wallis test followed by Dunn's Multiple Comparison Test). e) Quantification of the Phosphatidic Acid (PA) / PhosphatidyICholine (PC) ratio in EVs isolated from shControl, shRalA and shRalB cells (each dot represents one experiment; 3 independent experiments; One Way Anova permutation test followed by fdr multi-comparison permutation test; $\mathrm{fdr}<0,1)$. f) Model showing how RalA and RalB could control PLD1 localization on MVBs, thereby inducing the PA accumulation on MVBs, promoting MVB homeostasis and controlling exosome secretion. 
bioRxiv preprint doi: https://doi.org/10.1101/2020.07.10.196691; this version posted July 10, 2020. The copyright holder for this preprint (which was not certified by peer review) is the author/funder. All rights reserved. No reuse allowed without permission.
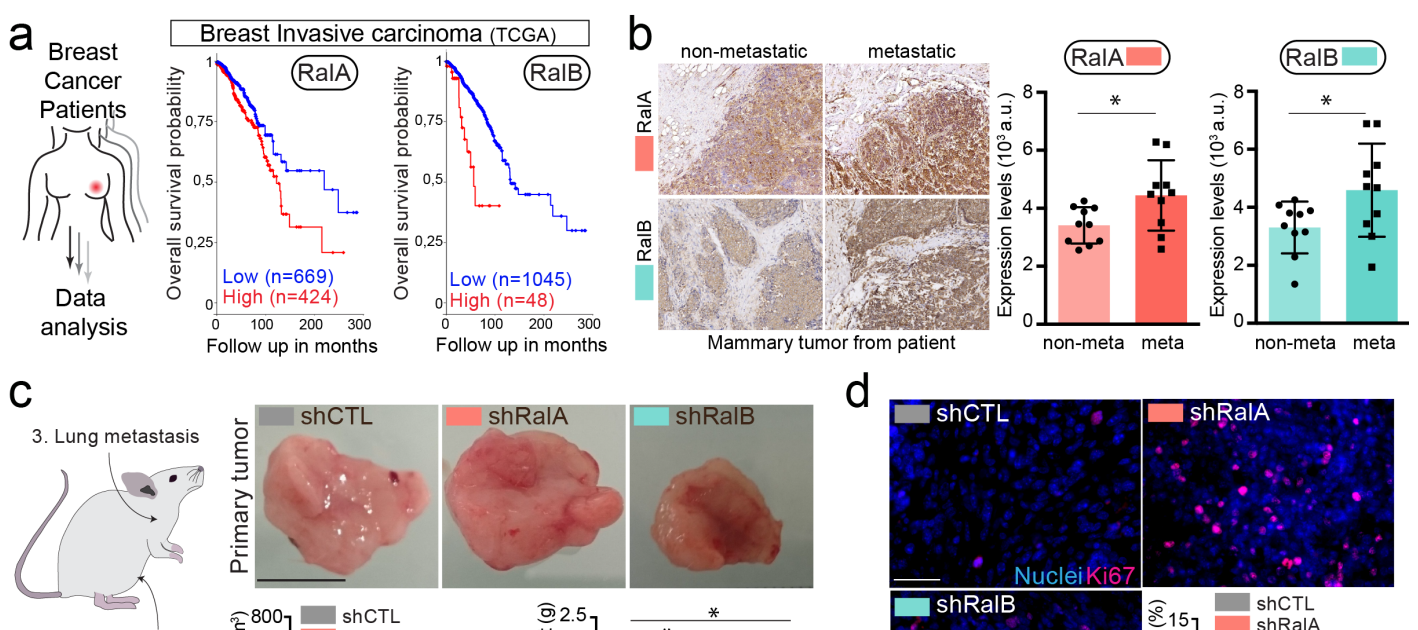

d

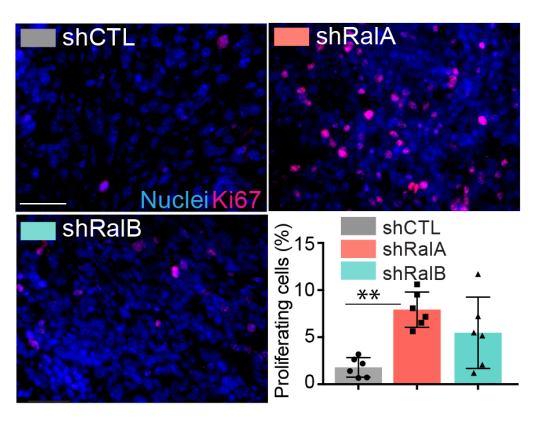

e

1. Intraductal grafts of $4 \mathrm{~T} 1$ cells
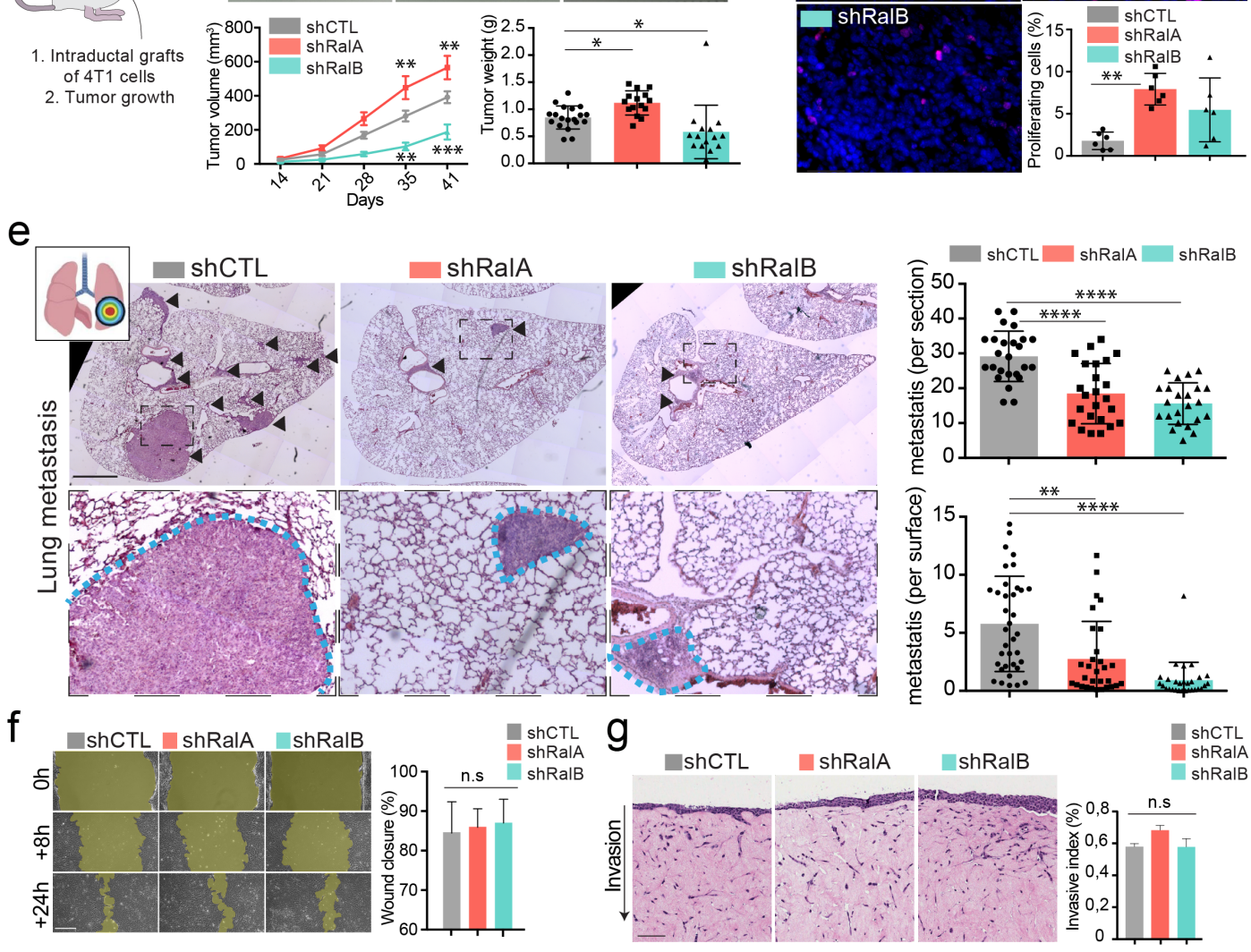

Fig. 3. RalA and RalB promote lung metastasis in a non cell autonomous fashion. a) Kaplan-Meier curve, obtained from TCGA 1097 cohort, showing the survival probability of patients with tumor breast invasive carcinoma having high or low RalA (pvalue: 5,15 e-03; pAdj: 1,35e-01) or RalB (pvalue: 1,77 e-05; pAdj: 5,99e-03) expression levels. b) Representative images of immunohistochemistry against RalA or RalB performed on mammary primary tumors from patients with or without metastasis. Scale bar: 500 $\mu \mathrm{m}$ Graphs represent automated scoring of DAB staining. Each dot represents one patient; 10 patients per group; Student t-test. c) Orthotopic injection of shControl, shRalA and shRalB 4T1 cells in syngenic mice. Representative images of primary tumors at day 41 . Scale bar: $1 \mathrm{~cm}$. Graphs showing the primary tumor growth over time (Left) and the primary tumor weight at day 41. Each dot represents one mouse. (Two independent experiments; Left: Two way Anova, Rlght: Kruskal-Wallis test followed by Bonferonni post Test). d) Representative confocal images of primary tumors stained with anti-Ki67 antibody. Scale bar: $50 \mu \mathrm{m}$. Graph indicates the per cent of Ki67 positive nuclei. Each dot represents one mouse. ( 6 mice taken from 2 independent experiments; Kruskal-Wallis test followed by Dunn's Multiple Comparison Test. e) Analysis of lung metastasis in mice from the orthotopic experiment presented in (c). Representative images of lung sections (Day 41) stained with hematoxilin eosin. Scale bar: 1mm. Graphs show the number of metastatic foci per section (upper, One Way Anova followed by Bonferroni's Multiple Comparison Test) and the metastatic surface per lung surface (lower; Kruskal-Wallis test followed by Dunn's Multiple Comparison Test). Each dot represents one section f) Pictures of wound healing closure at different time points. Scale bar: $150 \mu \mathrm{m}$. Graph represents the percentage of wound closure at $16 \mathrm{~h}$ ( 3 independent experiments; Kruskal-wallis test followed by Dunn's multiple comparison test). g) Pictures of $3 \mathrm{D}$ invasion assay after 15 days. Graph represents the invasive index. Scale bar: $100 \mu \mathrm{m}$. 
treated with a similar amount of EVs derived from shRalA or shRalB cells. Similarly, such EVs fail to disrupt adherent and tight junctions by contrast to EVs derived from $4 \mathrm{~T} 1$ control cells (Figure 4b) suggesting that EVs from RalA/B knockdown cells have reduced pro-permeability abilities. Therefore, depletion of RalA/B reduces secretion levels of EVs and leads to the secretion of EVs whose effect on vascular leakiness is hampered. The important observation that vascular permeability could be reduced upon depletion of RalA or RalB, and with a similar amount of EVs, prompted us to further dissect whether RalA or RalB could tune the priming of pre-metastatic niches.

RalA and RalB dependent EVs are pro-metastatic and lung tropic Here, we thus explored whether RalA and RalB synergistically impact the pro-metastatic functions of EVs by tuning their secretion levels as well as their content. Since on one hand RalA and RalB positively control the levels and the functionality of secreted tumor EVs (Figure 1 and 4a), and on the other hand they promote metastasis (Figure 3), we tested a direct impact of RalA/B-dependent EVs on the promotion of lung metastasis. For this, we decided to directly assess the role of $4 \mathrm{~T} 1 \mathrm{EVs}$ in priming lung metastatic niches in vivo, as previously described for other tumor EVs (Costa-Silva et al., 2015; Hoshino et al., 2015; Peinado et al., 2012; Zhou et al., 2014). Priming of lungs with control EVs significantly enhances lung metastasis over 14 days when compared to PBS (Figure 4c). In striking contrast, priming of mouse lungs with a similar number of EVs derived from Ral-depleted cells did not promote metastasis. This key experiment demonstrates that RalA/B confer pro-metastatic functions to EVs, in addition to controlling their secretion levels. Indeed, the decreased metastasis observed in absence of RalA/B can result from either drastically reduced EVs secretion or diminished pro-metastatic potential of EVs. To unravel why EVs from $\mathrm{RalA} / \mathrm{B}$ depleted cells are unable to promote metastasis, we first determined their capacity to efficiently reach the lungs and prime pre-metastatic niches by tracking the dissemination of fluorescently labeled EVs that were injected in the blood circulation of Balb/c mice. We found that one hour after injection 4T1 EVs mostly accumulate in the lungs, as well as the liver and brain (Figure $4 \mathrm{~d}$ and Supplementary Figure 6a). These three organs are the main metastatic organs of $4 \mathrm{~T} 1$ cells, and breast carcinoma, showing that the organotropism of 4T1 EVs mirrors the metastatic organotropism of their parental cells and further validates the relevance of our model to human pathology(Kaur et al., 2012; Lou et al., 2008). Through a careful analysis of cell types that internalize EVs in these conditions, we observed that 4T1 EVs mostly accumulate in endothelial cells, macrophages and fibroblasts of the lung parenchyma (Supplementary Figure 6b). Importantly, EVs derived from RalA or RalB depleted cells failed to efficiently reach the lungs, even though similar amounts were injected in all conditions (Figure 4d, e). Similar results were observed for EVs reaching the liver (Supplementary Figure $6 c)$. Hence, we can conclude at this stage that RalA/B control the pro-metastatic properties of EVs by tuning their ability to reach vascular regions and local parenchyma and effi- ciently reach metastatic organs, thereby modulating the formation of a pre-metastatic niche. The latter results raised the exciting hypothesis that metastasis impairment could be, in part, explained by a general defect in adhesion of circulating EVs at the vascular wall. We recently showed that EVs target specific vascular regions by first arresting at the surface of endothelial cells(Hyenne et al., 2019). We used two complementary models that allow careful tracking of single EVs and assessed early events of EVs internalization in endothelial cells. Using microfluidics, we found that internalization of 4T1 EVs within endothelial cells is decreased after one hour when they originate from RalA/B-depleted cells (Figure 4f). Similarly, upon tracking of fluorescent EVs injected in the circulation of zebrafish embryos, we observed that endothelial arrest/internalization of EVs from RalA/B knockdown cells is significantly hampered (Figure $4 \mathrm{~g}$ ). Altogether, these experiments suggest that RalA/B knockdown significantly reduced the adhesive properties of EVs to the endothelium, establishing a potential link with their failure to accumulate in mice lungs. Furthermore, our results support a model in which RalA/B GTPases, in addition to promoting EV secretion, also control the pro-metastatic function of these EVs, likely by modulating their content.

\section{RalA/B promote CD146 EV loading to efficiently colonize} lungs These functional experiments (Figure 4) suggest that the content of EVs can directly influence metastasis formation and that such content is likely to be impacted by RalA/B. Therefore, we carried out a careful and thorough molecular comparison of the cargo content of EVs derived from RalA/B-tuned cells. We first analyzed the RNA content of EVs using RNAseq and found that a large proportion of the RNAs present in EVs from shRal cells were different from the control (30-50per cent) (Figure 5a; Table 1). Accordingly, GO terms associated with mRNA enriched in each EV type showed important differences in biological processes, molecular function or cellular components (Supplementary Figure 7). In addition, EVs from shRalA cells differed from control or shRalB EVs in the nature of the RNA they contain, as shRalA EVs showed an important increase in noncoding RNA (Figure 5b). Overall, this experiment reveals that RalA/B have a profound impact on the content of RNA in 4T1 EVs. We further analyzed the protein content of 4T1 EVs by mass spectrometry. As shown in Figure 5c, 4T1 EVs contain a large number of proteins usually found in small EVs (77 of the top 100 proteins from Exocarta are found in 4T1 EVs; Table 2), such as tetraspanins, integrins, ESCRT proteins or small GTPases, such as RalA/B themselves. Unexpectedly, comparison of the proteome of EVs secreted by RalA or RalB knockdown cells did not reveal major differences, as no protein is exclusive to one type of EVs. Instead, a small proportion of proteins showed differential expression levels (Figure 5d; Table 2). Regarding their protein content, we noted that EVs from control cells are closer to EVs from shRalB cells (97 proteins with differential expression) than to EVs from shRalA cells (217 proteins with differential expression). We then focused on the five proteins over-expressed in EVs from shCtl cells compared to both 
bioRxiv preprint doi: https://doi.org/10.1101/2020.07.10.196691; this version posted July 10, 2020. The copyright holder for this preprint (which was not certified by peer review) is the author/funder. All rights reserved. No reuse allowed without permission.
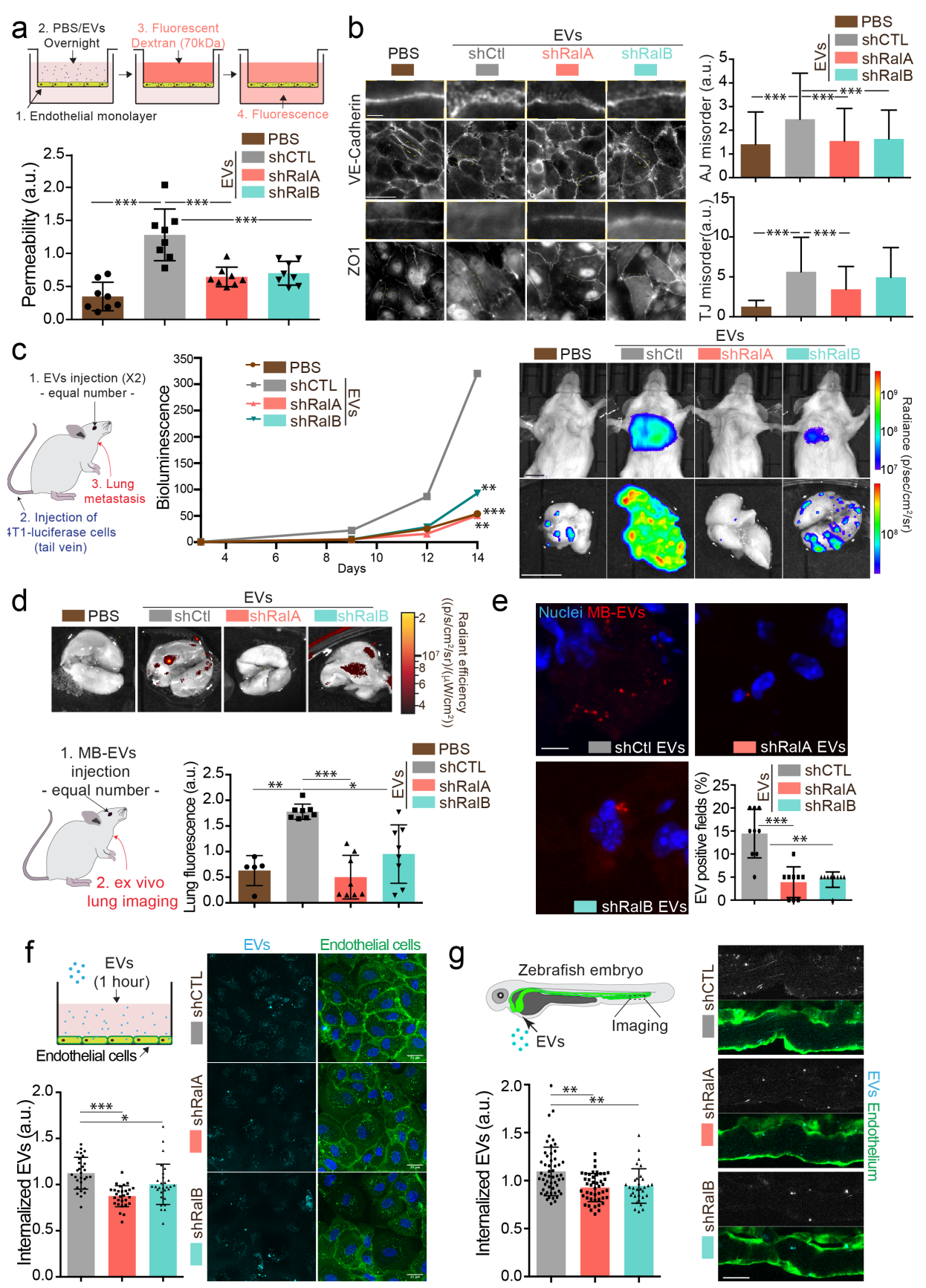

Fig. 4. RalA and RalB control lung tropism of pro-metastatic tumor EVs. a) Effect of a similar amount of EVs on HUVEC monolayer permeability in vitro. The graph represents the normalized amount of fluorescent dextran that crossed the endothelial barrier. Each dot represents one experiment (8 independent experiments; One Way Anova followed by Bonferroni's Multiple Comparison Test). b) Representative epifluorescence images of VE-cadherin (upper panels) and ZO1 (Lower panel) stainings on HUVECS cells treated with similar amounts of EVs. Scale bar: $20 \mu \mathrm{m}$; zoom: $2 \mu \mathrm{m}$. Graphs represent the disorganization of adherent (Three independent experiments; up; Kruskal-Wallis test followed by Dunn's Multiple Comparison Test) and tight (low; Kruskal-Wallis test followed by Dunn's Multiple Comparison Test) junctions. c) Metastasis priming experiment, Balb/c mice are first injected twice with tumor equal number of EVs (1,5x108 EVs), then intravenously with $4 \mathrm{~T} 1$ luciferase cells and metastasis is then followed over time. Graph shows metastasis progression over time in mice pre-injected with PBS, or with equal number of EVs from shControl, shRalA or shRalB cells (7-10 mice per group; merge of two independent experiments; Two way Anova followed by Bonferonni multiple comparison test; stars indicate statistically significant differences at day 14). Right: In vivo and ex vivo representative images of mice and lungs at day 14 . Scale bars: $1 \mathrm{~cm}$. d-e) Lung accumulation of equal number of fluorescent-labeled EVs (3 $108 \mathrm{EVs}$ ), from shControl, shRalA or shRalB cells injected intravenously. d) Representative ex vivo images and graph showing the total lung fluorescence $1 \mathrm{~h}$ post-injection. Each dot represents one mouse. (8 mice taken from 2 independent experiments; Kruskal-Wallis test followed by Dunn's Multiple Comparison Test. e) Representative confocal lung sections images and graph showing the percentage of EVs positive רา fields. Each dot represents one section (3 mice; Kruskal-Wallis test followed by Dunn's Multiple Comparison Test). Scale bar: $5 \mu \mathrm{m}$. $\mathrm{f}-\mathrm{g}$ ) Arrest and internalization of equal number of EVs from shControl, shRalA and shRalB cells on endothelial cells in vitro and in vivo. f) Representative confocal Z-stacks of equal number of EVs after $1 \mathrm{~h}$ or incubation with HUVEC monolayer. Scale bar: $25 \mu \mathrm{m}$. Each dot represents one field of view (each dot represents one field of view from 3 independent experiments; Kruskal-Wallis test followed by Dunn's Multiple Comparison Test). g) Representative confocal Z-stacks the caudal plexus of $\mathrm{Tg}$ (Fli1:GFP) zebrafish embryos, where GFP is expressed in the endothelium, injected with similar number of EVs and imaged right after injection. Each dot represents one zebrafish (31 to 53 embryos from 4 independent experiments; Kruskal-Wallis test followed by Dunn's Multiple Comparison Test). Scale bar: $20 \mu \mathrm{m}$. 
EVs from shRalA and EVs from shRalB cells. These proteins are CD146/MCAM, Clic4, Glypican 4, BDKRB2 and Abcg2. We verified the expression levels of CD146/MCAM, Clic4 and Glypican 4 by western blot of identical number of EVs (Figure 5e). While Clic4 and Glypican 4 are significantly under-expressed in EVs from shRalA or shRalB cells, the long isoform of CD146/MCAM (Supplementary Figure 8a) showed a significant decrease in EVs from shRalA cells, and a tendency to decrease in EVs from shRalB cells, which was confirmed by anti-CD146 ELISA (Supplementary Figure $8 \mathrm{~b}$ ). Altogether, content analysis reveals that depletion of either RalA or RalB deeply affects the EV RNA loading and changes the levels of several key proteins. We next interrogated whether the impact of RalA/B on the lung targeting and priming potential of EVs could be explained by its impact on the EV levels of MCAM/CD146.. MCAM/CD146 (also known as Mel-CAM, Muc18, S-endo1, Gicerin) is an adhesion receptor overexpressed in various cancer types, including breast cancer, where it was shown to promote invasion and tumor progression(Garcia et al., 2007; Zeng et al., 2011, 2012). In addition, MCAM/CD146 is present on endothelial cells where it mediates the adhesion of several cell types, including the transendothelial migration of monocytes(Bardin et al., 2009). Given, the known function of MCAM/CD146 in cell adhesion(Wang and Yan, 2013), we hypothesized that it may, at least in part, be responsible for the lung tropism defects observed with EVs derived from RalA/B-depleted cells. To test the involvement of MCAM/CD146 in EVs adhesion, we treated 4T1 EVs with an anti-mouse MCAM/CD146 blocking antibody before injection in zebrafish or mouse circulation. EVs pretreated with MCAM/CD146 blocking antibody failed to successfully arrest on endothelial walls of zebrafish embryos (Figure 5f) and inefficiently reached the lungs in our mouse model (Figure $5 \mathrm{~g}$ ). Therefore, inhibition of MCAM/CD146 phenocopies RalA/B knockdown. These results demonstrate that MCAM/CD146, whose presence at the surface of EVs is tuned by $\mathrm{RalA} / \mathrm{B}$, is, at least partly responsible of the adhesion and lung tropism of 4T1 EVs. It further explains why EVs from RalA knockdown cells, which have reduced levels of MCAM/CD146, fail to reach the lungs efficiently. The pro-metastatic role of MCAM/CD146 is further confirmed by the analysis of a human cohort of breast cancer showing that its high expression is associated with worsened prognosis (Figure 5h). Altogether, our work demonstrates that RalA/B, by controlling MVB homeostasis, promote the secretion CD146-enriched EVs, whose lung tropism sustains efficient metastasis (Figure 5i).

\section{Discussion}

The therapeutic limitations of breast cancer metastasis warrant a deeper understanding of its molecular machinery. Our findings highlight the exosome-mediated priming of metastatic niches by Ral GTPases as a critical requisite for lung metastasis during breast cancer progression. We show that RalA and RalB promote the secretion of exosomes by maintaining a high number of multi-vesicular bodies, likely through the PLD1-PA axis. Furthermore, we demonstrate that RalGTPases favor the secretion of exosomes that are enriched in CD146 and accumulate in metastatic organs, notably in lungs (Figure 5i). Finally, we show that high levels of RalA and RalB correlated with poor prognosis suggesting a unified mechanism for human breast cancer metastasis. This work, together with our previous study of RAL-1 in C. elegans(Hyenne et al., 2015), establishes Ral GTPases as major evolutionarily conserved mediators of exosome secretion. Our experiments suggest that RalA/B contribute to exosome secretion in several tumor cell lines, of different origins, implying that they might function pleiotropically over various cancers. These results further identify Ral inhibitors as new tools to inhibit exosome secretion in different contexts. Our results suggest that RalA/B and their effector PLD1 affect the levels of secreted exosomes by tuning the levels of cytoplasmic MVBs. While Ral GTPases, partially localized at the plasma membrane, could also affect microvesicle secretion, our data indicate that they mainly function in exosome biogenesis upstream of PLD1. Indeed, the absence of Ral (in both nematodes and mice), or the inhibition of PLD1 in 4T1 cells, drastically reduces the number of MVBs, which is accompanied by a decrease in secreted EVs. Similarly, a direct correlation between MVB density and levels of secreted EVs was recently suggested by studies showing that chemical or electric stimulation of MVB biogenesis results in increased EV secretion(Kanemoto et al., 2016; Yang et al., 2020). The formation of MVBs results from dramatic biochemical transformations of endosomes involving multiple protein and lipid switches(Huotari and Helenius, 2011; Scott et al., 2014). Understanding the steps at which RalA/B and PLD affect this endosome maturation program is critical and remains to be fully deciphered. Our results suggest that biogenesis of ILVs, which is a key step in MVB maturation and the initial phase of the exosome secretion pathway, is likely to be controlled by RalA/B. First, MVBs formed in total RAL-1 knock-out in C. elegans contained drastically less ILVs (Hyenne et al., 2015). Second, we show here that, knockdown of RalB, but not RalA, in 4T1 cells resulted in a significant decrease in ILV number. Differences between C. elegans and mouse data could result from an incomplete knockdown of RalA/B in $4 \mathrm{~T} 1$ cells or from a redundancy between RalA and RalB in ILV biogenesis. Our work further identifies PLD as the most likely effector acting downstream of Ral to control exosome secretion. Interestingly, while PLD2 was found to impact exosome secretion by governing ILV biogenesis in a different breast carcinoma cell line(Ghossoub et al., 2014), our data rather suggest that PLD1 controls exosome biogenesis in 4T1 cells. Indeed, PLD1 localizes on MVBs and its inhibition, but not the inhibition of PLD2, decreases MVB density. By contrast, PLD2 is essentially localized at the plasma membrane of 4T1 cells and its inhibition reduces EV secretion suggesting that PLD2 could rather promote microvesicle secretion in 4T1 cells. Therefore, we speculate that RalA/BPLD1 control ILV biogenesis in 4T1 cells. Alternatively, they could impact the homeostasis of a subclass of MVBs, for instance by controlling their stability or their degradation. The generation of PA by PLDs has been shown to affect multi- 
bioRxiv preprint doi: https://doi.org/10.1101/2020.07.10.196691; this version posted July 10, 2020. The copyright holder for this preprint (which was not certified by peer review) is the author/funder. All rights reserved. No reuse allowed without permission.
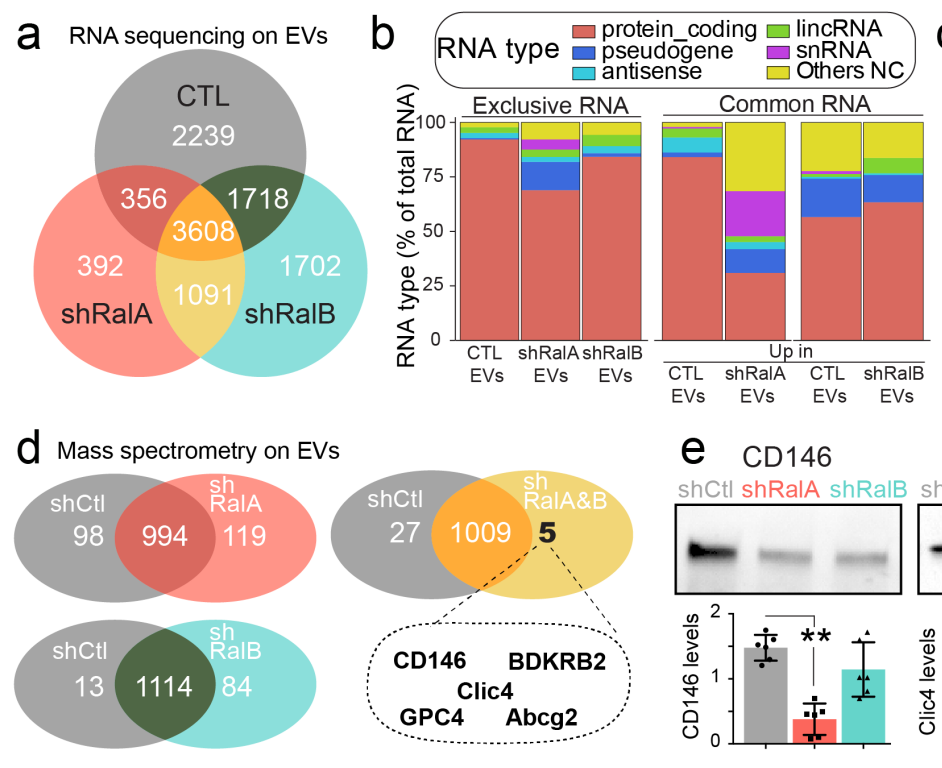

e $\quad$ CD146
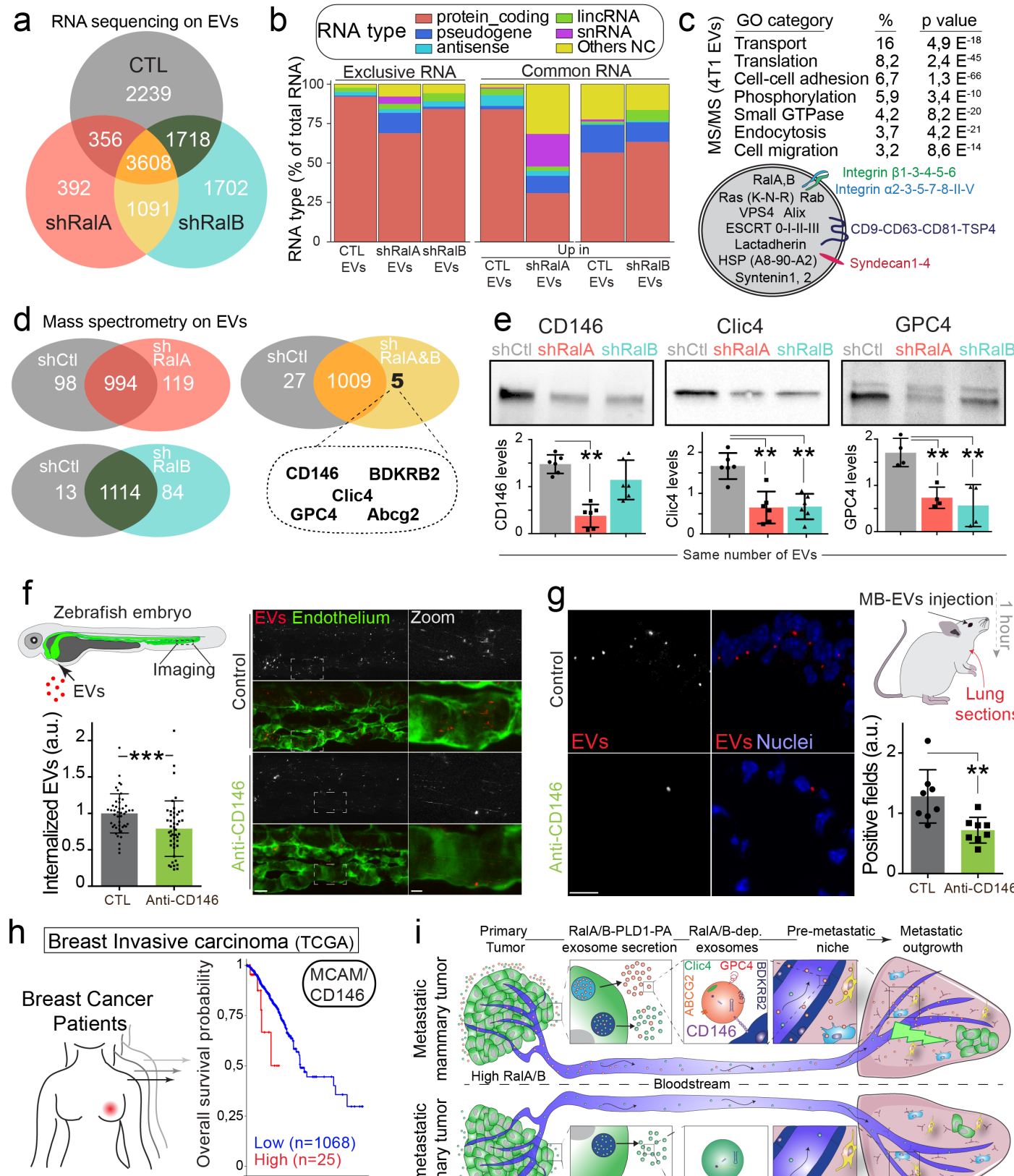

MB-EVs injection $\rightarrow$
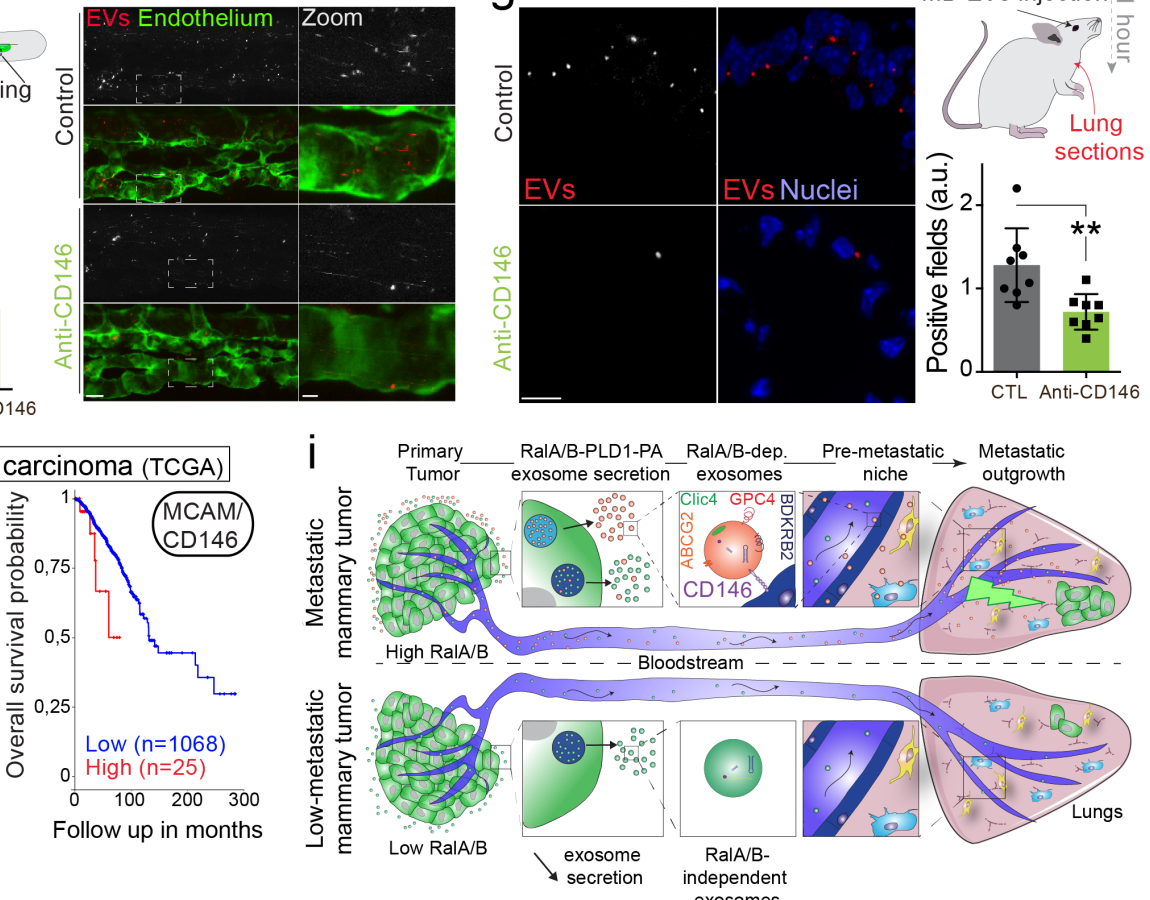

Fig. 5. CD146/MCAM is under-expressed in RalA/B knockdown EVs and mediates their lung tropism. a) Venn diagram representing the RNA present in the EVs isolated from shControl, shRalA or shRalB cells (with a minimum of 10 reads per sample; RNA sequencing performed in triplicate). b) Type of RNA associated identified in EVs isolated from shControl, shRalA or shRalB cells. Left: RNA exclusively present in one type of EVs. Right: enriched RNAs (log2 fold change $>2$; $p($ adj.) $<0,05$ ). c) GO terms of the proteins identified in EVs isolated from 4T1 cells by ultracentrifugation (100.000g pellet) and illustration of some proteins known to be present in EVs. d) Comparison of the protein content of EVs isolated from shControl, shRalA and shRalB cells. The venn diagram represents proteins having different expression levels (Mass spectrometry performed in triplicate; FDR< 1per cent). e) Analysis of the expression of CD146/MCAM, Clic4 and Glypican 4 in EVs isolated from shControl, shRalA and shRalB cells by western blots. Each dot represents one experiment (4 to 6 independent experiments; Kruskal-Wallis test followed by Dunn's Multiple Comparison Test). f-g) Arrest, internalization and organotropism of EVs treated with an anti-CD146 antibody and injected in the circulation of zebrafish embryos (f) or mouse (g). f) Representative confocal Z-stacks the caudal plexus of Tg(Fli1:GFP) zebrafish embryos, where GFP is expressed in the endothelium, injected with equal number of EVs and imaged right after injection. Scale bar: $20 \mu \mathrm{m}$; Zoom scale bar: $5 \mu \mathrm{m}$. Each dot represents one zebrafish (46 embryos from 4 independent experiments; Mann Whitney test). g) Representative confocal images of lung sections and graph showing the percentage of EVs positive fields. Scale bar: $10 \mu \mathrm{m}$. Each dot represents one mouse (8 mice from 2 independent experiments; Mann Whitney test). h) Kaplan-Meier curve, obtained from TCGA 1097 cohort, showing the survival probability of patients with tumor breast invasive carcinoma having high or low MCAM/CD146 expression levels (pvalue: 3,42 e-02; pAdj: 5,67e-01). i) Model describing the role of RalA/B dependent EVs in metastatic formation. 
ple steps of cancer progression(Bruntz et al., 2014; Roth and Frohman, 2018). Here, we show that the levels of PA of EVs are controlled by RalA/B, acting upstream of PLD, and thus validate initial observations suggesting that $\mathrm{PA}$ orchestrates MVB homeostasis and exosome secretion (Ghossoub et al., 2014). Another enzyme that is responsible for PA synthesis, diacylglycerol kinase $\alpha(\mathrm{DGK} \alpha)$, directly affects MVB concentration and exosome secretion in T lymphocytes (Alonso et al., 2005, 2011). However, DGK $\alpha$ seems to function antagonistically to PLDs, as it negatively regulates the formation of mature MVBs and the secretion of exosomes, suggesting the importance of tight spatiotemporal regulation of PA levels and probably of PA species on MVBs. PA is a pleiotropic bioactive lipid, which can activate or locally recruit specific proteins. It was shown to directly bind syntenin and induce negative membrane curvature, thereby favoring ILV budding and exosome secretion (Ghossoub et al., 2014; Kooijman et al., 2005). Independently of the precise mode of action of PA, further work is required to determine how the Ral-PLD1-PA axis is connected to other known machineries of ILV biogenesis. Priming of metastatic niches by (soluble or) EV-mediated factors takes central stages in cancer progression (Gao et al., 2019; Peinado et al., 2017) and identification of molecular machineries that underlie this condition could point to new therapeutic or diagnostic targets. Our study demonstrates that Ral GTPases enhance the formation of lung metastasis by promoting the secretion of exosomes within primary tumors. While the overexpression or the over-activation of RalA and RalB were previously described in several human cancers, including breast cancer ( $\mathrm{Li}$ et al., 2009), we now show that increased expression of RalA or RalB in primary tumor correlates with the formation of metastasis, in relevant mouse models and human patients. Interestingly, both Ral A and RalB can be detected in 4T1 EVs by mass spectrometry. This raises the exciting option to probe expression levels of RalA and RalB concomitantly in primary tumors and liquid biopsies as novel markers of bad prognosis. While the pro-tumoral activity of Ral GTPases was so far mostly attributed to their capacity to promote anchorage-independent cell growth (for RalA) or cell invasion (for RalB) (Yan and Theodorescu, 2018), we now show that Ral GTPases also have non-cell autonomous functions, and that these functions are important contributors to metastasis. Indeed, in 4T1 cells, depletion of either RalA or RalB alters the levels, content and functionality of secreted EVs, without decreasing cell migration or proliferation. Depending on the cell type or the biological process, RalA and RalB can display redundant, synergistic or even antagonist activities (Gentry et al., 2014). Since RalA and RalB mostly share similar phenotypes regarding EV secretion, content and function, they likely function in the same pathway. Interestingly, both Ral proteins appear to be essential for exosome secretion, revealing that their functions are not fully redundant. Therefore, both GTPases are required for the generation of a specific subpopulation of EVs with enhanced pro-metastatic properties. With this work, RalA and RalB add to the list of proteins known to control ex- osome secretion and to affect tumor progression, such as Rab27a (Bobrie et al., 2012; Kren et al., 2020; Peinado et al., 2012), Alix (Monypenny et al., 2018), syntenin (Das et al., 2019) and components of the ESCRT machinery (Mattissek and Teis, 2014). These studies demonstrate that the number of EVs secreted by a primary tumor is an essential element determining the efficiency of metastasis. However, it is important to keep in mind that all these proteins regulating EV trafficking, including RalA/B, contribute to tumor progression through both exosome dependent and exosome independent functions. For instance, Rab27a and Alix were recently shown to affect metastasis through both cellautonomous (by promoting invasion and EGFR activity, respectively) and non-cell autonomous function (by enhancing exosome secretion) (Kren et al., 2020; Monypenny et al., 2018). Similarly, we showed that RalA/B modulate the secretion of soluble molecules, such as WISP-1/CCN4, which is a known pro-metastatic factor likely to contribute to tumor progression (Ombrato et al., 2019). Altogether, despite pointing to additional functions of RAL GTPases, our study is the first to identify new molecular machinery from its function in $\mathrm{EV}$ biogenesis up to its pro-metastatic function in breast cancer lung metastasis. Priming of metastatic niches by EVs has, so far, mostly been attributed to increased levels of pro-metastatic EVs with pro-metastatic functions (Becker et al., 2016; Bobrie et al., 2012; Peinado et al., 2012). EV cargo molecules are thus key drivers of this condition and their identification is likely to unravel new therapeutic targets. In addition to controlling the levels of secreted EVs, we show that RalA/B affect their function by enhancing their capacity to induce endothelial permeability in vitro and premetastatic niches in vivo. These two observations could be linked, as RalA/B dependent EVs could promote endothelial permeability locally in the primary tumor or at distance in lungs, thereby favoring both tumor intravasation and extravasation. Content analysis revealed that RalA/B control the identity and levels of RNAs and proteins present in secreted EVs. Interestingly, Ras, which is known to activate RalA/B (Gentry et al., 2014), also controls the protein and RNA cargo of tumor EVs(Cha et al., 2015; Demory Beckler et al., 2013; McKenzie et al., 2016), although its effect on the levels of secreted EVs is unclear(Demory Beckler et al., 2013; McKenzie et al., 2016). As McKenzie and collaborators identified a MEK-ERK-Ago2 pathway downstream of Ras(McKenzie et al., 2016), it would be interesting to determine how this pathway connects with the Ral-PLD-PA axis described in our study/here. Among the few proteins significantly enriched in RalA/B dependent EVs, we identified CD146, a molecule known to modulate cell-cell adhesion(Wang and Yan, 2013). We showed, using functional inhibition, that CD146 present on pro-metastatic EVs controls their lung targeting efficiency thereby impacting their biodistribution and niche-promoting function. Accordingly, we and others show that high expression of CD146 correlates with poor prognosis in human breast carcinoma (Garcia et al., 2007; Zeng et al., 2012). CD146 functions as an adhesion molecule involved in homophilic and heterophilic interactions(Wang and Yan, 2013), 
promoting for instance monocyte transmigration(Bardin et al., 2009). CD146 can perform trans-homophilic interactions via its immunoglobulin-like extracellular domain(Taira et al., 1994, 2005). It also binds to extracellular matrix proteins or other transmembrane proteins, such as VEGFR2(Wang and Yan, 2013). Therefore, it is tempting to speculate that CD146 affects the biodistribution and organ targeting efficiency of circulating tumor EVs by mediating their interaction with specific ligands present on the luminal side of endothelial cells of metastatic organs. Other adhesion molecules, such as integrins and tetraspanins were shown to affect the biodistribution of tumor EVs and ultimately the formation of metastasis (Hoshino et al., 2015; Yue et al., 2015). Therefore, it is likely that the combination of these receptors at the surface of tumor EVs, combined with the differential expression of their ligands on endothelial cells throughout the organism will dictate their homing. More work will be needed to identify relevant endothelial ligands for circulating EV and develop inhibitory strategies to impair their arrest and uptake at metastatic sites. In addition, the presence of other cell types in the circulation, such as patrolling monocytes, which take up large amounts of circulating EVs, could also contribute to the accumulation of tumor EVs in specific organs (Hyenne et al., 2019; Plebanek et al., 2017). Finally, other factors, such as the vascular architecture and hemodynamic patterns could be involved (Follain et al., 2020; Hyenne et al., 2019) and the interplay between these mechanical cues and the surface repertoire of metastatic EVs should be a fertile ground for future research. Precisely dissecting the mechanisms by which tumor EVs reach specific organs would allow to understand the priming of premetastatic niches. Overall, our study identifies RalA/B GTPases as a novel molecular machinery that regulates the formation and shedding of pro-metastatic EVs and offers new potential targets (RalA/B and CD146) for developing new therapeutic strategies to impact the progression of metastatic breast cancer.

13

\section{Methods}

Cell culture The establishment of $4 \mathrm{~T} 1$ cell lines stably expressing shRNA against RalA, RalB, or a scramble sequence has been described previously (Hyenne et al., 2015). 4T1-Luciferase (RedLuc) cells were purchased from PerkinElmer. All 4T1 cell lines were cultured in RPMI-1640 medium, completed with 10per cent fetal bovine serum (FBS, Hyclone) and 1per cent penicillin-streptomycin (PS) (GIBCO). 4T1 shRNA cell lines were maintained in medium containing $1 \mu \mathrm{g} / \mathrm{ml}$ puromycin, except during experiments, and regularly checked for the stability of knockdown by western blots. Human Umbilical Vein Endothelial Cells (HUVEC) (PromoCell) were grown in ECGM (PromoCell) supplemented with a supplemental mix (PromoCell C-39215) and 1 per cent PS. Human A375 melanoma and human MDAMB-231 breast cancer (ATCC) cell lines were grown in high-glucose Dulbecco's modified Eagle's Medium (DMEM, Gibco Invitrogen Corporation) supplemented with 10per cent (FBS) and 1per cent PS. Human Panc-1 pancreatic adenocar- cinoma cell line was grown in RPMI 1640 supplemented with 10per cent FBS, and $50 \mu \mathrm{g} / \mathrm{ml}$ gentamicin sulfate (Gibco/Life Technologies). All cell lines were cultured in a humidified atmosphere containing 5per cent $\mathrm{CO} 2$ at $37^{\circ} \mathrm{C}$. Plasmid transfections: Cells at 50-70per cent confluency were transfected with $1 \mu \mathrm{g}$ of plasmid using JetPRIME (PolyPlus, Illkirch, France) according to the manufacturer's instructions. The following plasmids were used: pGFP-PLD1, pGFP-PLD2 (Corrotte et al., 2006), pLenti CMV:tdtomatoRalA and pLenti CMV:tdtomato-RalB. Drug treatment. Cells were incubated with the following drugs in the appropriate medium: RalA/B inhibitors BQU57 (10 $\mu \mathrm{M}$; Sigma) and RBC8 (10 $\mu$ M; Sigma), PLD1 inhibitor CAY10593 (10 $\mu \mathrm{M}$; Santa Cruz Biotechnology) or PLD2 inhibitor CAY10594 (10 $\mu \mathrm{M}$; Santa Cruz Biotechnology). Cells were treated for $18 \mathrm{~h}$ before processing for $\mathrm{EV}$ isolation or cell analysis.

qRT-PCR Analysis Total RNA was extracted from cells using TRI Reagent (Molecular Research Center) according to the manufacturer's instructions. For qRT-PCR, RNA was treated with DNase I and reverse transcribed using the High Capacity cDNA RT Kit. qRT-PCR was performed using the Power SYBR Green PCR Master Mix or TaqMan Gene Expression Master Mix using a 7500 Real Time PCR machine (Applied Biosystems). All compounds were purchased from Life Technologies (St Aubin, France). Data were normalized using a Taqman mouse probe against GADPH as endogenous control (4333764T, Life Technology) and fold induction was calculated using the comparative Ct method (-ddCt).

Western blot Cell or EV extracts were denatured in Laemmli buffer and incubated at $95^{\circ} \mathrm{C}$ for $10 \mathrm{~min} .10 \mu \mathrm{g}$ of protein extract (for cell lysates) or equal number of EVs (8.50x108 EVs per lane, measured by NTA) were loaded on 4per cent-20per cent polyacrylamide gels (Bio-Rad Laboratories, Inc.). The following antibodies were used: CD9 (Rat, 553758; BD Biosciences), RalA (mouse, 610221; BD Biosciences), RalB (mouse, 04037; Millipore), Glypican 4 (Rabbit, PA5-97801; Thermo Fisher Scientific), antibodies specifically recognizing the short and long isoforms of CD146 were previously described(Kebir et al., 2010), Clic4 (mouse, 135739; Santa Cruz Biotechnology), $\alpha$-tubulin (mouse, CP06; Millipore) and Secondary horseradish peroxidase -linked antibodies: anti-Rat (GE healthcare; NA935), anti-Mouse (GE healthcare; NA 931) and anti-rabbit (GE healthcare; NA934). Acquisitions were performed using a PXi system (Syngene). Intensities were measured using the Fiji software.

Elisa Elisa was performed according to the manufacture's instruction (RayBiotech) by loading equal number of EVs ( $7 \times 108$ - 9.5x109) per well ( 2 experiments in triplicate).

Electron microscopy Chemical fixation: Cells were fixed with 2,5per cent glutaraldehyde/2,0per cent paraformaldehyde (PFA) (Electron Microscopy Sciences) in $0.1 \mathrm{M} \mathrm{Ca}-$ codylate buffer at room temperature for $2 \mathrm{~h}$, then rinsed in 0.1M Cacodylate buffer (Electron Microscopy Sciences) and post-fixed with 1per cent OsO4 (Electron Microscopy Sciences) and 0.8per cent $\mathrm{K} 3 \mathrm{Fe}(\mathrm{CN}) 6$ (Sigma-Aldrich) for $1 \mathrm{~h}$ at $4^{\circ} \mathrm{C}$. Then, samples were rinsed in $0.1 \mathrm{M}$ Cacodylate buffer followed by a water rinse and stained with 1per cent uranyl 
acetate, overnight at $4^{\circ} \mathrm{C}$. The samples were stepwise dehydrated in Ethanol (50per cent, 70per cent 2x10min, 95per cent $2 \times 15 \mathrm{~min}$ and 100 per cent $3 \times 15 \mathrm{~min}$ ), infiltrated in a graded series of Epon (Ethanol100per cent /Epon 3/1, 1/1, 1h) and kept in Ethanol 100per cent /Epon 1/3 overnight. The following day, samples were placed in pure Epon and polymerized at $60^{\circ} \mathrm{C} .100 \mathrm{~nm}$ thin sections were collected in 200 copper mesh grids and imaged with a Philips CM12 transmission electron microscope operated at $80 \mathrm{kV}$ and equipped with an Orius 1000 CCD camera (Gatan). High-pressure freezing: HPF was performed using an HPF COMPACT 03 high pressure freezing machine (Wohlwend), using $3 \mathrm{~mm}$ diameter Aclar film disks (199um thickness), as cell carriers. Subsequent freeze substitution in acetone was performed using an automatic FS unit (Leica AFS), including 0.25per cent $\mathrm{OsO} 4$ staining, and Epon embedding. Sections were contrasted on grids with 1per cent uranyl acetate followed with 0,4per cent lead citrate (Sigma-Aldrich). Imaging was performed similarly to chemical fixation. The number of MVBs and lysosomes per surface of cytoplasm were quantified using the Fiji software. MVBs and lysosomes were distinguished based on their morphology: MVBs have one or more ILVs and lysosomes contain ILVs but are also electron dense and contain irregular membrane curls.

FACS analysis Confluent cells were incubated with $1 \mu \mathrm{M}$ Lysotracker Green DND 26 (L7526-Thermo Fischer) diluted in complete RPMI medium for 30 minutes at $37^{\circ} \mathrm{C}$. Cells were then detached by addition of TrypLE (12604021, ThermoFischer), washed in PBS 2per cent (v/v) FCS, and stained with $0.1 \mu$ M DAPI in PBS 2per cent (v/v) FCS immediately before analysis. Samples were processed on a Gallios Flow Cytometer (Beckman Coulter). Dead cells and doublets were excluded from analysis respectively by the selection of DAPI negative cells and co-analysis of integral vs time-of-flight side scatter signals. Data were analyzed on FlowJo software (BD Bioscience). Mean Fluorescence intensities (MFI) of lysotracker in each condition were normalized by performing a ratio with MFI of an unstained condition in the same channel.

Migration assays For 2D migration assays, 4T1 mammary tumor cells were plated on $35-\mathrm{mm}$ plastic dishes (6 well plates) and grown for 2 days until reaching 90per cent confluence. The cells were then grown for $16 \mathrm{~h}$ in serum-free medium before wounding of the monolayer by scraping from the middle of the plate. Cells were incubated in complete RPMI medium and sequential images of the wound were collected with a $10 \mathrm{X}$ objective at 0,8 and $24 \mathrm{~h}$ after wounding. Percentage of wound closure over time was analyzed and quantified using the Fiji software. 3D Organotypic invasion assays were conducted as previously described (Timpson et al., 2011; Vennin et al., 2017). Briefly, rat tail tendon collagen was extracted with $0.5 \mathrm{~mol} / \mathrm{L}$ acetic acid to a concentration of $2.5 \mathrm{mg} / \mathrm{ml}$. $8.4 \times 104$ telomerase immortalized fibroblasts (TIFs) were embedded into the neutralized collagen in the presence of $1 \times$ MEM and 8.8per cent FBS. Matrices were allowed to contract over a 12-day period in DMEM (1per cent P/S, 10per cent FBS). Following contraction TIFs were removed with puromycin $(2 \mu \mathrm{g} / \mathrm{ml})$ for 72 hours before $8 \times 1044 \mathrm{~T} 1$ cells were seeded on the contracted matrices and allowed to grow to confluence for 48 hours in RPMI (1per cent $\mathrm{P} / \mathrm{S}, 10$ per cent FBS). The matrices were then transferred to an air-liquid interface on a metal grid and cells allowed to invade for 15 days with media changes every 2 days. Following the invasion, organotypic matrices were fixed in 10per cent buffered formalin and processed for histochemical analysis. The invasive index was measured in 3 representative fields of view per matrix with three matrices per replicate for three replicates. Invasive Index $=($ Number of cells $>200 \mu \mathrm{m}$ depth $) /($ Cells on top of the matrix $)$

In vitro permeability assay Transwell filter inserts (pore size $1.0 \mu \mathrm{m}, 12 \mathrm{~mm}$ diameter, polyester membrane, Corning, New York, USA) were coated with fibronectin $(10 \mu \mathrm{g} / \mathrm{ml}$; Sigma). Then, HUVECs were seeded $(0.3 \times 106$ cells/well $)$ and grown on transwell filters for $48 \mathrm{~h}$ until reaching confluency. Confluent monolayers of HUVEC cells were treated with similar amounts (10-100ug) of 4T1-EVs, PBS (as a negative control) or with $100 \mathrm{ng} / \mathrm{ml} \mathrm{TNF}-\alpha$ (as a positive control) overnight. FITC-dextran (MW 70,000; Sigma) was added to the top well at $25 \mathrm{mg} / \mathrm{ml}$ for $20 \mathrm{~min}$ at $37^{\circ} \mathrm{C}$, and fluorescence was measured in the bottom well using a fluorescence plate reader (Berthold Tris Star 2; $485 \mathrm{~nm}$ excitation and $520 \mathrm{~nm}$ emission). Cells were washed for 3 times and were fixed for immunofluorescence (described below).

Secretome analysis Cell culture supernatants were collected and centrifuged for 15 minutes at $300 \mathrm{~g}$. Supernatants were incubated with Mouse XL Cytokine Array membranes (RD Systems) according to the manufacturers' instructions. Three independent experiments were performed. Intensities were measured using the Fiji software.

in vitro proliferation assay Briefly, cells were seeded in 96well plates at the density of 2000 cells per well with $200 \mu \mathrm{l}$ of complete culture medium and cultured for 24,48 and $72 \mathrm{~h}$ at $37^{\circ} \mathrm{C}$. Culture medium without cells was used as the blank control group. To avoid the edge effect, the peripheral wells were filled with sterile PBS. For the proliferation test, a total of $20 \mu \mathrm{l}$ MTS solution was added to each well, followed by incubation for $2 \mathrm{~h}$ at $37^{\circ} \mathrm{C}$. Optical density was measured at 490nm using a Berthold Tristar device.

EVs isolation and characterization Cells were cultured in EV depleted medium (obtained by overnight ultracentrifugation at $100,000 \mathrm{~g}$, using a Beckman, XL-70 centrifuge with a $70 \mathrm{Ti}$ rotor) for $24 \mathrm{~h}$ before supernatant collection. The extracellular medium was concentrated using a Centricon Plus70 centrifugal filter (10k; Millipore) and EVs were isolated by successive centrifugation at $4{ }^{\circ} \mathrm{C}$ : 15 minutes at $300 \mathrm{~g}, 10$ minutes at 2,000 g, 30 minutes at $10,000 \mathrm{~g}$ and 70 minutes at $100,000 \mathrm{~g}$ (using a Beckman XL-70 centrifuge with a SW28 rotor). EVs pellets were washed in PBS, centrifuged again at $100,000 \mathrm{~g}$ for 70 minutes, resuspended in PBS and stored at $4^{\circ} \mathrm{C}$. For all functional experiments, EVs were used immediately after isolation or stored overnight at $4{ }^{\circ} \mathrm{C}$ and injected the next day. For content analysis, EVs were frozen at $-80^{\circ} \mathrm{C}$. After EV isolation, EVs numbers and size distribution were measured by NTA using a ZetaView (Particle Metrix, 
Meerbusch, Germany). For in vivo mouse experiments, EVs were isolated the using the iZON qEV2 size exclusion column (Izon science, Cambridge MA) according to the manufacturer's instructions. After rinsing the columns with PBS, $2 \mathrm{ml}$ of concentrated extracellular medium were applied on top of a qEV column (Izon Science) and $6 \mathrm{ml}$ fractions were collected. For organotropism experiments, four EV-rich fractions (F2, F4, F6, and F8) were pooled, then ultracentrifuged for $1 \mathrm{~h}$ at $100,000 \times \mathrm{g}, 4^{\circ} \mathrm{C}$ with a SW28 rotor in a Beckman XL-70 centrifuge or concentrated using an Amicon Ultra-4 $10 \mathrm{kDa}$ centrifugal filter device (Merck Millipore). Pellets were resuspended in $500 \mu \mathrm{L}$ PBS. For priming experiment, the most EV-rich fraction was used (F4). For fluorescent labeling, isolated EVs were incubated with MemBright-Cy3 or Cy5 (Collot et al., 2018) at 200nM (zebrafish) and 500nM (mice) (final concentration) in PBS for 30 minutes at room temperature in the dark. Labeled EVs were then rinsed in $15 \mathrm{ml}$ of PBS, centrifuged at $100,000 \mathrm{~g}$ with a SW28 rotor in a Beckman XL-70 centrifuge and pellets were resuspended in $50 \mu \mathrm{L}$ PBS. EVs were used immediately after isolation or stored for a maximum of one night at $4^{\circ} \mathrm{C}$ before use.

Mass spectrometry-based proteomic experiments Sample preparation of EVs Proteins. $20 \mathrm{mg}$ samples were denatured at $95{ }^{\circ} \mathrm{C}$ for $5 \mathrm{~min}$ in Laemmli buffer and concentrated in one stacking band using a 5per cent SDS-PAGE gel. The gel was fixed with 50per cent ethanol/3per cent phosphoric acid and stained with colloidal Coomassie Brilliant Blue. The gel bands were cut, washed with ammonium hydrogen carbonate and acetonitrile, reduced and alkylated before trypsin digestion (Promega). The generated peptides were extracted with 60 per centacetonitrile in 0.1 per cent formic acid followed by a second extraction with 100per cent acetonitrile. Acetonitrile was evaporated under vacuum and the peptides were resuspended in $10 \mu \mathrm{L}$ of $\mathrm{H} 20$ and 0.1 per cent formic acid before nanoLC-MS/MS analysis. NanoLCMS/MS analysis. NanoLC-MS/MS analyses were performed on a nanoACQUITY Ultra-Performance LC system (Waters, Milford, MA) coupled to a Q-Exactive Plus Orbitrap mass spectrometer (ThermoFisher Scientific) equipped with a nanoelectrospray ion source. The solvent system consisted of 0.1 per cent formic acid in water (solvent A) and 0.1per centformic acid in acetonitrile (solvent B). Samples were loaded into a Symmetry C18 precolumn $(0.18$ x $20 \mathrm{~mm}, 5 \mu \mathrm{m}$ particle size; Waters) over $3 \mathrm{~min}$ in 1 per cent solvent $\mathrm{B}$ at a flow rate of $5 \mu \mathrm{L} / \mathrm{min}$ followed by reverse-phase separation (ACQUITY UPLC BEH130 C18, $200 \mathrm{~mm} \times 75 \mu \mathrm{m}$ id, 1.7 $\mu \mathrm{m}$ particle size; Waters) using a linear gradient ranging from 1per cent to 35per cent of solvent B at a flow rate of $450 \mathrm{~nL} / \mathrm{min}$. The mass spectrometer was operated in datadependent acquisition mode by automatically switching between full MS and consecutive MS/MS acquisitions. Survey full scan MS spectra (mass range 300-1800) were acquired in the Orbitrap at a resolution of $70 \mathrm{~K}$ at $200 \mathrm{~m} / \mathrm{z}$ with an automatic gain control (AGC) fixed at 3.106 and a maximal injection time set to $50 \mathrm{~ms}$. The ten most intense peptide ions in each survey scan with a charge state 2 were selected for fragmentation. MS/MS spectra were acquired at a resolution of $17,5 \mathrm{~K}$ at $200 \mathrm{~m} / \mathrm{z}$, with a fixed first mass at $100 \mathrm{~m} / \mathrm{z}$, AGC was set to 1.105 , and the maximal injection time was set to $100 \mathrm{~ms}$. Peptides were fragmented by higher-energy collisional dissociation with a normalized collision energy set to 27. Peaks selected for fragmentation were automatically included in a dynamic exclusion list for $60 \mathrm{~s}$. All samples were injected using a randomized and blocked injection sequence (one biological replicate of each group plus pool in each block). To minimize carry-over, a solvent blank injection was performed after each sample. EVs mass spectrometry was performed in triplicate. Data interpretation. Raw MS data processing was performed using MaxQuant software1 v1.6.7.0 (Cox et al., 2014). Peak lists were searched against a database including Mus musculus protein sequences extracted from SwissProt (09-10-2019; 17 007 sequences, Taxonomy ID $=10$ 090). MaxQuant parameters were set as follows: MS tolerance set to $20 \mathrm{ppm}$ for the first search and $5 \mathrm{ppm}$ for the main search, MS/MS tolerance set to $40 \mathrm{ppm}$, maximum number of missed cleavages set to 1, Carbamidomethyl (C) set as a fixed modification, Oxidation (M) and Acetyl (Protein N-term) set as variable modifications. False discovery rates (FDR) were estimated based on the number of hits after searching a reverse database and were set to 1 per cent for both peptide spectrum matches (with a minimum length of seven amino acids) and proteins. All other MaxQuant parameters were set as default. Protein intensities were used for label free quantification. The imputation of the missing values (DetQuantile imputation) and differential data analysis were performed using the open-source ProStaR software(Wieczorek et al., 2017). A Limma moderated $\mathrm{t}$-test was applied on the dataset to perform differential analysis. The adaptive Benjamini-Hochberg procedure was applied to adjust the p-values and FDR values under 1per cent were achieved. Complete dataset has been deposited to the ProteomeXchange Consortium via the PRIDE partner repository5 with the dataset identifier PXD020180(Deutsch et al., 2020).

RNA sequencing EV pellets were treated with proteinase $\mathrm{K}(0.05 \mu \mathrm{g} / \mu \mathrm{l})$ for $10 \mathrm{~min}$ at $37 \mathrm{C}^{\circ}$. Roche Cocktail Inhibitor was then added to the sample for $10 \mathrm{~min}$ at room temperature followed by incubation at $85 \mathrm{C}^{\circ}$ for $5 \mathrm{~min}$. Samples were then incubated with RNase A $(0.5 \mu \mathrm{g} / \mu \mathrm{l})$ for $20 \mathrm{~min}$ at $37 \mathrm{C}^{\circ}$ to degrade unprotected RNA. Total RNAs of isolated EVs was extracted using TRI Reagent (Molecular Research Center). Total RNA Sequencing libraries were prepared with SMARTer® Stranded Total RNA-Seq Kit v2 - Pico Input Mammalian (TaKaRa) according to the manufacturer's instructions. Libraries were pooled and sequenced (paired-end $2 * 75 b p$ ) on a NextSeq500 using the NextSeq 500/550 High Output Kit v2 according to the manufacturer's instructions (Illumina, San Diego, CA, USA). Raw sequencing data generated by the Illumina NextSeq500 instrument were mapped to the mouse reference genome using the hisat2 software(Kim et al., 2015). For every sample, quality control was carried out and assessed with the NGS Core Tools FastQC (http://www.bioinformatics.babraham.ac.uk/projects/fastqc/). Read counts were generated with the htseq-count tool of the 
Python package HTSeq (Anders et al., 2015). Differential analysis was performed by the DESEQ2 (Love et al., 2014) package of the Bioconductor framework. Detection of significantly up- and down-regulated genes between pairs of conditions based on their $\log 2 \mathrm{FC}$ and functional enrichment analyses were performed using STRING v11 (Szklarczyk et al., 2019). EVs RNA sequencing was performed in triplicate.

Lipidomics EVs were extracted with $2 \mathrm{ml}$ of chloroform $/$ methanol $2 / 1 \mathrm{v} / \mathrm{v}$ and $1 \mathrm{ml}$ water, sonicated for $30 \mathrm{~s}$, vortexed, and centrifuged. Lower organic phase was transferred to a new tube, the upper aqueous phase was re-extracted with $2 \mathrm{ml}$ chloroform. Organic phases were combined and evaporated to dry. Lipid extracts were resuspended in $50 \mu \mathrm{L}$ of eluent A. Synthetics internals lipid standards (PA 14:1/17:0, PC 17:0/14:1 and PS 17:0/17:0) from Avanti Polar Lipids was added. LC-MS/MS (MRM mode) analyses were performed with a MS model QTRAP® 6500 (ABSciex) coupled to an LC system (1290 Infinity II, Agilent). Analyses were achieved in the negative (PA) and in positive (PC) mode; nitrogen was used for the curtain gas (set to 20), gas 1 (set to 20) and gas 2 (set to1 0). Needle voltage was at 4, 500 ot 5, $500 \mathrm{~V}$ without needle heating; the declustering potential was adjusted set at $172 \mathrm{~V}$ or $+40 \mathrm{~V}$. The collision gas was also nitrogen; collision energy is set to 46 or $+47 \mathrm{eV}$. The dwell time was set to $30 \mathrm{~ms}$. Reversed phase separations were carried out at $50{ }^{\circ} \mathrm{C}$ on a Luna C8 $150 \times 1$ mm column, with $100 \AA$ pore size, $5 \mu \mathrm{m}$ particles (Phenomenex). Eluent A was isopropanol/CH3OH/H2O (5/1/4) +0.2 per cent formic acid +0.028 per cent NH3 and eluent $\mathrm{B}$ was isopropanol+0.2per cent formic acid +0.028 per cent $\mathrm{NH}$. The gradient elution program was as follows: 0-5 min, $30-50$ per cent B; $5-30$ min, $50-80$ per cent $\mathrm{B}$; $31-41 \mathrm{~min}$, 95 per cent $B ; 42-52$ min, 30 per cent $B$. The flow rate was set at $40 \mu \mathrm{L} / \mathrm{min} ; 15 \mu \mathrm{L}$ sample volumes were injected. The areas of LC peaks were determined using MultiQuant software (v3.0, ABSciex) for PA and PC quantification. EVs lipid analysis was performed in triplicate.

Animal experiments All animals were housed and handled according to the guidelines of INSERM and the ethical committee of Alsace, France (CREMEAS) (Directive 2010/63/EU on the protection of animals used for scientific purposes). Animal facility agreement number: C67-482-33. Experimental license for mice: Apafis 4707-2016032416407780; experimental license for zebrafish: Apafis 16862-2018121914292754. Mouse experiments: 6-8 weeks-old female BalB/c mice (Charles River) were used in all experiments. Orthotopic breast tumor experiments: Syngenic $\mathrm{BalB} / \mathrm{c}$ mice were injected in the left fourth mammary gland with 250.000 4T1 mammary tumor cells stably expressing either scramble control shRNA, RalA shRNA, or RalB shRNA and diluted in $50 \mu \mathrm{l}$ PBS. When tumors became palpable, tumor volume was assessed by caliper measurements using the formula (width $2 \times$ length) $/ 2(\mathrm{~mm} 3)$ twice a week for 41 days. At the endpoint of the experiment, tumors and lungs were harvested, weighted and fixed in formaldehyde. Alternatively, organs were embedded in OCT and frozen at $-80^{\circ} \mathrm{C}$. In this case, lungs were inflated with
OCT before dissection. Priming experiments: Mice were injected retro-orbitally with $1.5 \times 108 \mathrm{EVs}$ isolated from 4T1shControl, shRalA and shRalB cells. Two injections of EVs were performed two days apart. PBS was used as a negative control. Subsequently, 4T1-luciferase cells (90.000) were injected via tail vein one day after EV pre-conditioning. After cells injection, the extent of lung metastasis was measured every 3 days for 12 days using non-invasive imaging with IVIS Lumina III (Perkin Elmer). In brief, a Dluciferin solution (purchased from Perkin Elmer and used at $150 \mathrm{mg} / \mathrm{kg}$, according to manufacturer's instructions) was injected intraperitoneally to the isofluorane (Zoetis) anesthetized mice. $5 \mathrm{~min}$ after luciferin injection, a bioluminescence image was acquired with an IVIS Lumina III (Perkin Elmer) imaging system and then analyzed using the Living Image software (Perkin Elmer). The rate of total light emission of the lung metastatic area was calculated and expressed as radiance photons counted during the whole acquisition time $(5 \mathrm{~min})$ and normalized to the initial radiance photon (photon/second/cm2/sr) measured immediately after 4T1- luciferase cells injection for each mouse $(\mathrm{t} 0)$. EV biodistribution. Mice were injected via retro-orbital venous sinus with 1-4 × 108 MemBright-Cy3-labelled EVs freshly isolated from 4T1-shControl, shRalA and shRalB cells. PBS was used as a negative control. Mice were sacrificed $1 \mathrm{~h}$ post-injection to quantify the fluorescence intensity of the organs ex-vivo with IVIS Lumina III (Perkin Elmer).Average of fluorescent photons per lung were quantify as radiant efficiency [photon $\left./ \mathrm{second} / \mathrm{cm}^{2} / \mathrm{sr}\right] /\left[\mu \mathrm{W} / \mathrm{cm}^{2}\right]$. For experiment testing the role of CD146 in EV biodistribution, isolated EVs were incubated with CD146 blocking antibody (EPR3208; Abcam; $12 \mu \mathrm{g} / \mathrm{ml}$ ) for $30 \mathrm{~min}$ at room temperature before injection. Zebrafish experiments: At $48 \mathrm{~h}$ post-fertilization (hpf), $\operatorname{Tg}$ (Fli1 :GFP) zebrafish embryos were dechorionated and mounted in 0.8 per cent low melting point agarose pad containing $650 \mathrm{mM}$ of tricaine (ethyl-3-aminobenzoatemethanesulfonate). Embryos were injected in the duct of $\mathrm{Cu}-$ vier with 27,6 $\mathrm{nL}$ of Membright Cy5- labeled EVs (at 1010 $\mathrm{EVs} / \mathrm{ml}$ ) freshly isolated from 4T1-shControl, shRalA and shRalB cells with a Nanoject microinjector 2 (Drummond) under a M205 FA stereomicroscope (Leica), using microforged glass capillaries ( 25 to $30 \mathrm{~mm}$ inner diameter) filled with mineral oil (Sigma). Embryos were imaged with confocal right after injection. For experiment testing the role of CD146, 4T1isolated EVs were incubated with CD146 blocking antibody $(12 \mu \mathrm{g} / \mathrm{ml})$ for $30 \mathrm{~min}$ at room temperature before injection.

Tissue section and staining Mouse lungs were incubated overnight in 4per cent PFA, dehydrated in 100per cent ethanol for $24 \mathrm{~h}$, embedded in paraffin, cut in $7 \mu \mathrm{m}$ thick sections, dewaxed and rehydrated with 100per cent Toluene (2 washes of $15 \mathrm{~min}$ ) then incubated in 100per cent-70per cent alcohol solutions (10 min each) followed by final staining with hematoxylin (Surgipath) for $5 \mathrm{~min}$ and washing with tap water. Sections were further processed with differentiation solution (1per cent $\mathrm{HCl}$ in absolute ethanol, for $7 \mathrm{~s}$ ), followed by washing under tap water for $10 \mathrm{~min}$. Sections were then 
incubated in eosin (Harris) for $10 \mathrm{~s}$, rinsed and dehydrated in 70per cent - 100per cent alcohol baths with rapid dips in each bath before a final wash in toluene for $15 \mathrm{~min}$ and embedded in Eukitt solution (Sigma). 2 random distanced sections taken in each of the 5 lung were analyzed for each mouse. Stitching imaging was performed using an AxioImager (Zeiss) with a 10x objective. Metastatic surfaces and whole lung surfaces were measured using the Fiji software.

Caspase 3/7 assay Mouse tumor samples stored at $-80^{\circ} \mathrm{C}$ are disrupted in a buffer containing Tris $\mathrm{HCl} \mathrm{pH} \mathrm{7.5,} \mathrm{50mM,}$ $\mathrm{NaCl} 150 \mathrm{mM}$, NP40 1per cent + Protease Inhibitors cocktail (Complete from Roche) in the presence of 4 zirconium beads, using the Precellis system (Bertin instruments) with 2 pulses (10") at $5000 \mathrm{rpm}$. Protein concentration was measured using Bradford kit (BioRad) and $5 \mu \mathrm{g}$ was analyzed using the Caspase 3/7 glo kit (Promega) according to manufacturer's instructions. Photons production generated by the luciferase was measured using a luminometer (Berthold Tris Star 2).

Immunofluorescence For immunofluorescence on cultured cells, cells were fixed with 4per cent PFA for $15 \mathrm{~min}$, permeabilized in PBS-Triton 0.1per cent (Sigma) for $10 \mathrm{~min}$ and incubated in 5 per cent normal goat serum for $1 \mathrm{~h}$. The following primary antibodies were used: ZO-1 (Rabbit, 61-7300; Thermo Fisher Scientific), VE-Cadherin (mouse, 348502; BioLegend), CD63 (mouse, D623-3; MBL), RalA (mouse, 610221; BD), RalB (mouse, 04037; Millipore). The following secondary antibodies were used: goat antimouse/rat/rabbit coupled with Alexa Fluor 488, Alexa 555, or Alexa 647 (Invitrogen). Cells were mounted with DAPI-containing Vectashield (Vector Laboratories). For immunofluorescence on tissue sections, tissues were cut in $7 \mu \mathrm{m}$ thick sections, dewaxed for paraffin-embedded tissues and air-dried for frozen tissues. Sections were incubated first in 5 per cent normal goat serum for $2 \mathrm{~h}$ in a humidified container. The following antibodies were used: CD31 (Mouse, 37-0700; Thermo Fisher Scientific), S100A4 A gift from Nona Ambartsumian (Institut for Cancer Biology, Copenhagen, DK-2100, Denmark.), F4/80 (Rat, ab6640; abcam), rabbit monoclonal antibody against Ki67 (Rabbit, RM-9106S0; Thermo Fisher Scientific) and caspase-3 (Mouse, 966S1; Cell Signaling Technology). Secondary antibodies were similar to the ones used with cells. Nuclei were stained with DAPI (Sigma).

Imaging and Analysis Imaging on fixed samples. Tissue and cell sections were imaged with a Zeiss Imager Z2 with a 40X objective (N.A. 1.4) or with an SP5 confocal (Leica) with a $63 \mathrm{X}$ objective (N.A. 1.25). Image analysis and processing were performed using the Fiji software. For endothelial adherent and tight junction analysis, 10 random junctions were analyzed per image (5 images per sample) measuring junction width. For Ki67 and Caspase 3 imaging, 15 random fields of view were quantified per sample. For EVs imaging, 40 to 60 random fields of view were imaged on 3 to 4 sections per mouse. Live-cell imaging. For live-cell imaging, cells were seeded on $3.5 \mathrm{~cm}$ diameter glass-bottom dishes (MatTek Corporation, Ashland, MA) pre-coated with fibronectin $\left(10 \mu \mathrm{g} / \mathrm{ml}\right.$; Sigma). Nuclei were labeled with NucBlue ${ }^{\mathrm{TM}}$
Live Ready Probe (Life Technologies, Grand Island, NY). In some experiments, cells were incubated with Lysotracker Deep Red (Thermo Fisher Scientific) at $1 \mu \mathrm{M}$ for $30 \mathrm{~min}$ before imaging. Cells were imaged by confocal microscopy (SP5, Leica) equipped with a thermostated chamber at $37^{\circ} \mathrm{C}$ with 5per cent $\mathrm{CO} 2$. Image analysis and processing were performed using the Fiji software. HUVEC cells were seeded in fibronectin $(10 \mu \mathrm{g} / \mathrm{ml}$; Sigma) pre-coated glass bottom culture chambers (LabTek I, Dutscher 055082. Confluent cells were incubated with $2 \times 108$ MemBright-labeled EVs in EVfree medium for $1 \mathrm{~h}$. Nucleus were labeled using NucBlue ${ }^{\mathrm{TM}}$ (Life Technologies, Grand Island, NY). Cells were imaged by confocal microscopy (SP5 Leica) in a thermostated chamber at $37^{\circ} \mathrm{C}$ with 5 per cent $\mathrm{CO} 2$. Zebrafish imaging: Confocal imaging was performed on the caudal plexus of zebrafish embryos right after injection with an inverted TCS SP5 with HC PL APO 20X/0,7 IMM Corr CS objective (Leica). Image analysis and processing were performed using the Fiji software.

Human samples Human databases: Kaplan-Meier survival curves and statistical analysis of overall survival and gene expression was assessed on the TCGA breast invasive carcinoma cohort (1097 patients) using data generated by the TCGA Research Network: https://www.cancer.gov/tcga. Immunohistochemistry: Paraffin sections of $4 \mu \mathrm{m}$ from metastasic and non-metastasic breast tumours were obtained from CRB-Tumorothèque of the Institut de Cancérologie de l'Ouest (ICO, Saint-Herblain, France). Immunohistochemsitry was performed using RalA (BD Transduction 610222, 1/100) and RalB (Sigma WH0005899, 1/400) antibodies on MicroPICell facility (Nantes, France) Citrate buffer pH6 was used for antigen retrieval $20 \mathrm{~min}$ à $96^{\circ} \mathrm{C}$ (Target Retrieval solution low $\mathrm{pH}$, Dako) and $\mathrm{DAB}$ and Hematoxylin staining were revealed using ImPath detection kit (DAB OB Sensitive Detection Kit, ImPath). Whole slides were scanned on Hamamatsu scanner using Nanozoomer Digital Pathology software. Automated computer quantification of DAB staining in perinuclear zones (brown intensity measurement) after automatic nuclei detection with hematoxylin staining in the whole biopsies was performed using Qupath open source software for digital pathology image analysis (Bankhead et al., 2017) on MicroPICell platform (Nantes, France). Quantification was further confirmed by manual blinded arbitrary scoring of DAB brown intensity in tumoral zones was performed using a score of 1 for low staining to score of 3 for intense staining.

Statistical analyses. All results were confirmed in at least two independent experiments. Statistical significance of results was analyzed using the GraphPad Prism program version 5.04. The Shapiro-Wilk normality test was used to confirm the normality of the data. The statistical difference of Gaussian data sets was analyzed using the Student unpaired two-tailed $t$ test, with Welch's correction in case of unequal variances and the one-way ANOVA test followed by a Bonferonni multiple comparison post-test was used for multiple data comparison. For data not following a Gaussian distribution, the Mann-Whitney test was used, and the Kruskal- 
Wallis test followed by Dunn's Multiple Comparison posttest was used for multiple data comparison. Two Way Anova was used to compare more than 1 parameters followed by Bonferonni post-test. For analyzing data containing only 3 measurements, One Way Anova permutation test followed pairwise permutation test with false detection rate (fdr) correction, using R software (version 3.6.2) was used. Illustrations of these statistical analyses are displayed as the mean + - standard deviation (SD). p-values smaller than 0.05 were considered as significant. *, $\mathrm{p}<0.05, * *, \mathrm{p}<0.01, * * *, \mathrm{p}<$ $0.001, * * * *, \mathrm{p}<0.0001$.

Acknowledgements We thank all members of the Goetz Lab for helpful discussions, in particular Florent Colin for careful reading, as well as Gregory Khelifi and Camille Hergott for animal care. We thank the CRB-Tumorothèque of the Institut de Cancérologie de l'Ouest (ICO, Saint-Herblain, France) and the Cellular and Tissular Imaging Core Facility of Nantes University MicroPICell (SFR-Santé, Nantes, France). We thank Mayeul Collot and Andrey Klymchenko (UMR 7021) for providing the MemBright dye and Alexandre Detappe (INSERM U1109) for assistance on TCGA data analysis. This work was supported by a fellowship from IDEX (University of Strasbourg) and ARC (Association pour le Recherche sur le Cancer) to S.G.; by grants from La Ligue contre le Cancer, Canceropole Grand-Est, INCa (PLBIO19-291), Plan Cancer (Nanotumor) and Roche to J.G.G.; and by institutional funds from University of Strasbourg and INSERM to JGG, and ANR (to CC, French Proteomics Infrastructure ProFI, ANR-10-INBS-08-03; to NV, ANR-19-CE44-0019). The Metabolome Bordeaux facility was supported by the grant MetaboHUB-ANR-11-INBS0010. PT and KM were supported by Suttons, Sydney Catalyst, NHMRC, Cancer Council NSW, Cancer Institute NSW and by an Avner Pancreatic Cancer Foundation and Len Ainsworth Pancreatic Research Funds.

Author Contributions SG performed most experiments, with help from AL (IF, WB), NO (IF) and AK (sectioning). BM conducted microfluidics and zebrafish experiments. Electron microscopy was performed by VH, IB, CS, AMH and CR. FD and CC conducted MS experiments and analysis. NP and RC performed RNA sequencing and analysis with contribution from SH. FG did FACS experiments and analysis. LF performed the lipidomics analysis with contribution from NV. KM and PT performed 3D migration assays. MBC provided CD146 antibodies and expertise. GAG and JGa conducted IHC experiments on human samples. OL was involved in molecular biology, cell line generation, mice experiments and data analysis. JGG supervised and designed the project. VH performed cell biology, microscopy and animal experiments and designed the project. JGG (with help from $\mathrm{VH}$ ) was responsible for funding the project. JGG and $\mathrm{VH}$ wrote the manuscript with insights from all authors.

\section{Bibliography}

Alonso, R., Rodríguez, M.C., Pindadolh, J., Merino, E., Mérida, I., and Izquierdo, M. (2005). Diacylglycerol kinase $\alpha$ regulates the secretion of lethal exosomes bearing Fas lig- and during activation-induced cell death of $\mathrm{T}$ lymphocytes. Journal of Biological Chemistry.

Alonso, R., Mazzeo, C., Rodriguez, M.C., Marsh, M., FraileRamos, A., Calvo, V., Avila-Flores, A., Merida, I., and Izquierdo, M. (2011). Diacylglycerol kinase $\alpha$ regulates the formation and polarisation of mature multivesicular bodies involved in the secretion of Fas ligand-containing exosomes in T lymphocytes. Cell Death and Differentiation.

Anders, S., Pyl, P.T., and Huber, W. (2015). HTSeq-A Python framework to work with high-throughput sequencing data. Bioinformatics.

Bankhead, P., Loughrey, M.B., Fernández, J.A., Dombrowski, Y., McArt, D.G., Dunne, P.D., McQuaid, S., Gray, R.T., Murray, L.J., Coleman, H.G., et al. (2017). QuPath: Open source software for digital pathology image analysis. Scientific Reports.

Baran, J., Baj-Krzyworzeka, M., Weglarczyk, K., Szatanek, R., Zembela, M., Barbasz, J., Czupryna, A., Szczepanik, A., and Zembala, M. (2010). Circulating tumour-derived microvesicles in plasma of gastric cancer patients. Cancer Immunology, Immunotherapy 59, 841-850.

Bardin, N., Blot-Chabaud, M., Despoix, N., Kebir, A., Harhouri, K., Arsanto, J.P., Espinosa, L., Perrin, P., Robert, S., Vely, F., et al. (2009). CD146 and its soluble form regulate monocyte transendothelial migration. Arteriosclerosis, Thrombosis, and Vascular Biology 29, 746-753.

Becker, A., Thakur, B.K., Weiss, J.M., Kim, H.S., Peinado, H., and Lyden, D. (2016). Extracellular Vesicles in Cancer: Cell-to-Cell Mediators of Metastasis. Cancer Cell 30, 836-848.

Bobrie, A., Krumeich, S., Reyal, F., Recchi, C., Moita, L.F., Seabra, M.C., Ostrowski, M., and Théry, C. (2012). Rab27a supports exosome-dependent and -independent mechanisms that modify the tumor microenvironment and can promote tumor progression. Cancer Research 72, 4920-4930.

Bruntz, R.C., Lindsley, C.W., and Brown, H.A. (2014). Phospholipase D signaling pathways and phosphatidic acid as therapeutic targets in cancer. Pharmacological Reviews.

Cha, D.J., Franklin, J.L., Dou, Y., Liu, Q., Higginbotham, J.N., Beckler, M.D., Weaver, A.M., Vickers, K., Prasad, N., Levy, S., et al. (2015). KRAS-dependent sorting of miRNA to exosomes. ELife 4, 1-22. Collot, M., Ashokkumar, P., Anton, H., Boutant, E., Faklaris, O., Galli, T., Mély, Y., Danglot, L., and Klymchenko, A.S. (2018). MemBright: a family of red to near-infrared fluorescent membrane probes for advanced cellular imaging and neuroscience.

Corrotte, M., Chasserot-Golaz, S., Huang, P., Du, G., Ktistakis, N.T., Frohman, M.A., Vitale, N., Bader, M.F., and Grant, N.J. (2006). Dynamics and function of phospholipase $\mathrm{D}$ and phosphatidic acid during phagocytosis. Traffic 7, 365-377.

Costa-Silva, B., Aiello, N.M., Ocean, A.J., Singh, S., Zhang, H., Thakur, B.K., Becker, A., Hoshino, A., Mark, M.T., Molina, H., et al. (2015). Pancreatic cancer exosomes initiate pre-metastatic niche formation in the liver. Nature Cell Biology 1-7.

Cox, J., Hein, M.Y., Luber, C.A., Paron, I., Nagaraj, N., 
and Mann, M. (2014). Accurate Proteome-wide Label-free Quantification by Delayed Normalization and Maximal Peptide Ratio Extraction, Termed MaxLFQ. Molecular Cellular Proteomics.

Das, S.K., Sarkar, D., Emdad, L., and Fisher, P.B. (2019). MDA-9/Syntenin: An emerging global molecular target regulating cancer invasion and metastasis (Elsevier Inc.).

Demory Beckler, M., Higginbotham, J.N., Franklin, J.L., Ham, A.-J., Halvey, P.J., Imasuen, I.E., Whitwell, C., Li, M., Liebler, D.C., and Coffey, R.J. (2013). Proteomic analysis of exosomes from mutant KRAS colon cancer cells identifies intercellular transfer of mutant KRAS. Molecular Cellular Proteomics: MCP 12, 343-355.

Deutsch, E.W., Bandeira, N., Sharma, V., Perez-Riverol, Y., Carver, J.J., Kundu, D.J., García-Seisdedos, D., Jarnuczak, A.F., Hewapathirana, S., Pullman, B.S., et al. (2020). The ProteomeXchange consortium in 2020: Enabling "big data" approaches in proteomics. Nucleic Acids Research.

Follain, G., Herrmann, D., Harlepp, S., Hyenne, V., Osmani, N., Warren, S.C., Timpson, P., and Goetz, J.G. (2020). Fluids and their mechanics in tumour transit: shaping metastasis. Nature Reviews Cancer. Galindo-Hernandez, O., VillegasComonfort, S., Candanedo, F., González-Vázquez, M.C., Chavez-Ocaña, S., Jimenez-Villanueva, X., Sierra-Martinez, M., and Salazar, E.P. (2013). Elevated concentration of microvesicles isolated from peripheral blood in breast cancer patients. Archives of Medical Research 44, 208-214.

Gao, Y., Bado, I., Wang, H., Zhang, W., Rosen, J.M., and Zhang, X.H.F. (2019). Metastasis Organotropism: Redefining the Congenial Soil. Developmental Cell 49, 375-391.

Garcia, S., Dalès, J.P., Charafe-Jauffret, E., CarpentierMeunier, S., Andrac-Meyer, L., Jacquemier, J., Andonian, C., Lavaut, M.N., Allasia, C., Bonnier, P., et al. (2007). Poor prognosis in breast carcinomas correlates with increased expression of targetable CD146 and c-Met and with proteomic basal-like phenotype. Human Pathology 38, 830-841.

Gentry, L.R., Martin, T.D., Reiner, D.J., and Der, C.J. (2014). Ral small GTPase signaling and oncogenesis: More than just 15minutes of fame. Biochimica et Biophysica Acta 1843, 2976-2988.

Ghossoub, R., Lembo, F., Rubio, A., Gaillard, C.B., Bouchet, J., Vitale, N., Slavík, J., Machala, M., and Zimmermann, P. (2014). Syntenin-ALIX exosome biogenesis and budding into multivesicular bodies are controlled by ARF6 and PLD2. Nature Communications 5, 3477.

Hoshino, A., Costa-Silva, B., Shen, T.-L., Rodrigues, G., Hashimoto, A., Tesic Mark, M., Molina, H., Kohsaka, S., Di Giannatale, A., Ceder, S., et al. (2015). Tumour exosome integrins determine organotropic metastasis. Nature 1-19.

Huotari, J., and Helenius, A. (2011). Endosome maturation. EMBO Journal.

Hyenne, V., Apaydin, A., Rodriguez, D., Spiegelhalter, C., Hoff-Yoessle, S., Diem, M., Tak, S., Lefebvre, O., Schwab, Y., Goetz, J.G., et al. (2015). RAL-1 controls multivesicular body biogenesis and exosome secretion. The Journal of Cell Biology 211, 27-37.

Hyenne, V., Lefebvre, O., and Goetz, J.G.J.G. (2017). Going live with tumor exosomes and microvesicles. Cell Adhesion and Migration 11, 173-186.

Hyenne, V., Ghoroghi, S., Collot, M., Bons, J., Follain, G., Harlepp, S., Mary, B., Bauer, J., Mercier, L., Busnelli, I., et al. (2019). Studying the Fate of Tumor Extracellular Vesicles at High Spatiotemporal Resolution Using the Zebrafish Embryo. Developmental Cell 48, 554-572.e7.

Jiang, H., Luo, J.Q., Urano, T., Franke, P., Lu, Z., Foster, D.A., and Feig, L.A. (1995). Involvement of ral GTPase in v-Src-induced phospholipase D activation. Nature.

Jung, T., Castellana, D., Klingbeil, P., Cuesta Hernández, I., Vitacolonna, M., Orlicky, D.J., Roffler, S.R., Brodt, P., and Zöller, M. (2009). CD44v6 dependence of premetastatic niche preparation by exosomes. Neoplasia (New York, N.Y.) 11, 1093-1105.

Kanemoto, S., Nitani, R., Murakami, T., Kaneko, M., Asada, R., Matsuhisa, K., Saito, A., and Imaizumi, K. (2016). Multivesicular body formation enhancement and exosome release during endoplasmic reticulum stress. Biochemical and Biophysical Research Communications 1-7.

Kaplan, R.N., Riba, R.D., Zacharoulis, S., Bramley, A.H., Vincent, L., Costa, C., MacDonald, D.D., Jin, D.K., Shido, K., Kerns, S. a, et al. (2005). VEGFR1-positive haematopoietic bone marrow progenitors initiate the pre-metastatic niche. Nature 438, 820-827.

Kaur, P., Nagaraja, G.M., Zheng, H., Gizachew, D., Galukande, M., Krishnan, S., and Asea, A. (2012). A mouse model for triple-negative breast cancer tumor-initiating cells (TNBC-TICs) exhibits similar aggressive phenotype to the human disease. BMC Cancer 12, 120.

Kebir, A., Harhouri, K., Guillet, B., Liu, J.W., FoucaultBertaud, A., Lamy, E., Kaspi, E., Elganfoud, N., Vely, F., Sabatier, F., et al. (2010). CD146 short isoform increases the proangiogenic potential of endothelial progenitor cells in vitro and in vivo. Circulation Research.

Kim, D., Langmead, B., and Salzberg, S.L. (2015). HISAT: A fast spliced aligner with low memory requirements. Nature Methods.

Klumperman, J., and Raposo, G. (2014). The complex ultrastructure of the endolysosomal system. Cold Spring Harbor Perspectives in Biology 6, 1-22.

Kooijman, E.E., Chupin, V., Fuller, N.L., Kozlov, M.M., De Kruijff, B., Burger, K.N.J., and Rand, P.R. (2005). Spontaneous curvature of phosphatidic acid and lysophosphatidic acid. Biochemistry. Kren, N., Michaud, D., Bagchi, S., Greene, K., and Pylayeva-Gupta, Y. (2020). Rab27a plays a dual role in metastatic propensity of pancreatic cancer. Scientific Reports.

Lewis, J.A., Scott, S.A., Lavieri, R., Buck, J.R., Selvy, P.E., Stoops, S.L., Armstrong, M.D., Brown, H.A., and Lindsley, C.W. (2009). Design and synthesis of isoform-selective phospholipase D (PLD) inhibitors. Part I: Impact of alternative halogenated privileged structures for PLD1 specificity. Bioorganic and Medicinal Chemistry Letters.

Li, T.T., Alemayehu, M., Aziziyeh, A.I., Pape, C., Pampillo, M., Postovit, L., Mills, G.B., Babwah, A. V, and Bhattacharya, M. (2009). $\beta$-Arrestin / Ral Signaling Regulates 
Lysophosphatidic Acid - Mediated Migration and Invasion of Human Breast Tumor Cells. Molecular Cancer Research 7, 1064-1077. Logozzi, M., De Milito, A., Lugini, L., Borghi, M., Calabrò, L., Spada, M., Perdicchio, M., Marino, M.L., Federici, C., Iessi, E., et al. (2009). High levels of exosomes expressing CD63 and caveolin-1 in plasma of melanoma patients. PLoS ONE 4.

Lou, Y., Preobrazhenska, O., Auf Dem Keller, U., Sutcliffe, M., Barclay, L., McDonald, P.C., Roskelley, C., Overall, C.M., and Dedhar, S. (2008). Epithelial-Mesenchymal Transition (EMT) is not sufficient for spontaneous murine breast cancer metastasis. Developmental Dynamics.

Love, M.I., Huber, W., and Anders, S. (2014). Moderated estimation of fold change and dispersion for RNA-seq data with DESeq2. Genome Biology.

Luo, J.Q., Liu, X., Frankel, P., Rotunda, T., Ramos, M., Flom, J., Jiang, H., Feig, L. a, Morris, a J., Kahn, R. a, et al. (1998). Functional association between Arf and RalA in active phospholipase D complex. Proceedings of the National Academy of Sciences of the United States of America 95, 3632-3637.

Mathieu, M., Martin-Jaular, L., Lavieu, G., and Théry, C. (2019). Specificities of secretion and uptake of exosomes and other extracellular vesicles for cell-to-cell communication. Nature Cell Biology 21, 9-17. Mattissek, C., and Teis, D. (2014). The role of the endosomal sorting complexes required for transport (ESCRT) in tumorigenesis. Molecular Membrane Biology 31, 111-119.

McKenzie, A.J., Hoshino, D., Hong, N.H., Cha, D.J., Franklin, J.L., Coffey, R.J., Patton, J.G., and Weaver, A.M. (2016). KRAS-MEK Signaling Controls Ago2 Sorting into Exosomes. Cell Reports 15, 978-987. Monypenny, J., Milewicz, H., Flores-Borja, F., Weitsman, G., Cheung, A., Chowdhury, R., Burgoyne, T., Arulappu, A., Lawler, K., Barber, P.R., et al. (2018). ALIX Regulates Tumor-Mediated Immunosuppression by Controlling EGFR Activity and PD-L1 Presentation. Cell Reports.

van Niel, G., D'Angelo, G., and Raposo, G. (2018). Shedding light on the cell biology of extracellular vesicles. Nature Reviews Molecular Cell Biology.

Ombrato, L., Nolan, E., Kurelac, I., Mavousian, A., Bridgeman, V.L., Heinze, I., Chakravarty, P., Horswell, S., Gonzalez-Gualda, E., Matacchione, G., et al. (2019). Metastatic-niche labelling reveals parenchymal cells with stem features. Nature 572, 603-608.

Ostrowski, M., Carmo, N.B., Krumeich, S., Fanget, I., Raposo, G., Savina, A., Moita, C.F., Schauer, K., Hume, A.N., Freitas, R.P., et al. (2010). Rab27a and Rab27b control different steps of the exosome secretion pathway. Nature Cell Biology 12, 19-30; sup pp 1-13.

Oxford, G., Owens, C.R., Titus, B.J., Foreman, T.L., Herlevsen, M.C., Smith, S.C., and Theodorescu, D. (2005). RalA and RalB: Antagonistic relatives in cancer cell migration. Cancer Research 65, 7111-7120.

Peinado, H., Alečković, M., Lavotshkin, S., Matei, I., Costa-Silva, B., Moreno-Bueno, G., Hergueta-Redondo, M., Williams, C., García-Santos, G., Ghajar, C.M., et al. (2012).
Melanoma exosomes educate bone marrow progenitor cells toward a pro-metastatic phenotype through MET. Nature Medicine 18, 883-891.

Peinado, H., Zhang, H., Matei, I.R., Costa-Silva, B., Hoshino, A., Rodrigues, G., Psaila, B., Kaplan, R.N., Bromberg, J.F., Kang, Y., et al. (2017). Pre-metastatic niches: organ-specific homes for metastases. Nature Reviews Cancer 17, 302-317.

Plebanek, M.P., Angeloni, N.L., Vinokour, E., Li, J., Henkin, A., Martinez-Marin, D., Filleur, S., Bhowmick, R., Henkin, J., Miller, S.D., et al. (2017). Pre-metastatic cancer exosomes induce immune surveillance by patrolling monocytes at the metastatic niche. Nature Communications 8. Roth, E., and Frohman, M.A. (2018). Proliferative and metastatic roles for Phospholipase D in mouse models of cancer. Advances in Biological Regulation.

Scott, C.C., Vacca, F., and Gruenberg, J. (2014). Endosome maturation, transport and functions. Seminars in Cell Developmental Biology 31, 2-10.

Scott, S.A., Selvy, P.E., Buck, J.R., Cho, H.P., Criswell, T.L., Thomas, A.L., Armstrong, M.D., Arteaga, C.L., Lindsley, C.W., and Brown, H.A. (2009). Design of isoform-selective phospholipase D inhibitors that modulate cancer cell invasiveness. Nature Chemical Biology 5, 108-117.

Szklarczyk, D., Gable, A.L., Lyon, D., Junge, A., Wyder, S., Huerta-Cepas, J., Simonovic, M., Doncheva, N.T., Morris, J.H., Bork, P., et al. (2019). STRING v11: Proteinprotein association networks with increased coverage, supporting functional discovery in genome-wide experimental datasets. Nucleic Acids Research.

Taira, E., Takaha, N., Taniura, H., Kim, C.H., and Miki, N. (1994). Molecular cloning and functional expression of gicerin, a novel cell adhesion molecule that binds to neurite outgrowth factor. Neuron. Taira, E., Kohama, K., Tsukamoto, Y., Okumura, S., and Miki, N. (2005). Gicerin/CD146 is involved in neurite extension of NGFtreated PC12 cells. Journal of Cellular Physiology.

Timpson, P., McGhee, E.J., Erami, Z., Nobis, M., Quinn, J.A., Edward, M., and Anderson, K.I. (2011). Organotypic collagen I assay: A malleable platform to assess cell behaviour nin a 3-dimensional context. Journal of Visualized Experiments.

Tominaga, N., Kosaka, N., Ono, M., Katsuda, T., Yoshioka, Y., Tamura, K., Lötvall, J., Nakagama, H., and Ochiya, T. (2015). Brain metastatic cancer cells release microRNA181c-containing extracellular vesicles capable of destructing blood-brain barrier. Nature Communications 6, 6716.

Treps, L., Edmond, S., Harford-Wright, E., Galan-Moya, E.M., Schmitt, A., Azzi, S., Citerne, A., Bidère, N., Ricard, D., and Gavard, J. (2016). Extracellular vesicle-transported Semaphorin3A promotes vascular permeability in glioblastoma. Oncogene 35, 2615-2623.

Vennin, C., Chin, V.T., Warren, S.C., Lucas, M.C., Herrmann, D., Magenau, A., Melenec, P., Walters, S.N., Del Monte-Nieto, G., Conway, J.R.W., et al. (2017). Transient tissue priming via ROCK inhibition uncouples pancreatic cancer progression, sensitivity to chemotherapy, and metas- 
tasis. Science Translational Medicine.

Vitale, N., Mawet, J., Camonis, J., Regazzi, R., Bader, M.F., and Chasserot-Golaz, S. (2005). The small GTPase RalA controls exocytosis of large dense core secretory granules by interacting with ARF6-dependent phospholipase D1. Journal of Biological Chemistry 280, 29921-29928.

Wang, Z., and Yan, X. (2013). CD146, a multi-functional molecule beyond adhesion. Cancer Letters. Wang, D., Sun, H., Wei, J., Cen, B., and DuBois, R.N. (2017). CXCL1 is critical for premetastatic niche formation and metastasis in colorectal cancer. Cancer Research 77, 3655-3665.

Wang, Q., Ni, Q., Wang, X., Zhu, H., Wang, Z., and Huang, J. (2015). High expression of RAB27A and TP53 in pancreatic cancer predicts poor survival. Medical Oncology.

Wieczorek, S., Combes, F., Lazar, C., Gianetto, Q.G., Gatto, L., Dorffer, A., Hesse, A.M., Couté, Y., Ferro, M., Bruley, C., et al. (2017). DAPAR ProStaR: Software to perform statistical analyses in quantitative discovery proteomics. Bioinformatics.

Yan, C., and Theodorescu, D. (2018). RAL GTpases: Biology and potential as therapeutic targets in cancer. Pharmacological Reviews.

Yan, C., Liu, D., Li, L., Wempe, M.F., Guin, S., Khanna, M., Meier, J., Hoffman, B., Owens, C., Wysoczynski, C.L., et al. (2014). Discovery and characterization of small molecules that target the GTPase Ral. Nature 515, 443-447.

Yang, Z., Shi, J., Xie, J., Wang, Y., Sun, J., Liu, T., Zhao, Y., Zhao, X., Wang, X., Ma, Y., et al. (2020). Large-scale generation of functional mRNA-encapsulating exosomes via cellular nanoporation. Nature Biomedical Engineering.

Yue, S., Mu, W., Erb, U., and Zöller, M. (2015). The tetraspanins CD151 and Tspan8 are essential exosome components for the crosstalk between cancer initiating cells and their surrounding. Oncotarget 6, 2366-2384.

Zago, G., Veith, I., Singh, M.K., Fuhrmann, L., De Beco, S., Remorino, A., Takaoka, S., Palmeri, M., Berger, F., Brandon, N., et al. (2018). Ralb directly triggers invasion downstream ras by mobilizing the wave complex. ELife 7, 1-23.

Zeng, G.F., Cai, S.X., and Wu, G.J. (2011). Up-regulation of METCAM/MUC18 promotes motility, invasion, and tumorigenesis of human breast cancer cells. BMC Cancer 11, 113. Zeng, Q., Li, W., Lu, D., Wu, Z., Duan, H., Luo, Y., Feng, J., Yang, D., Fu, L., and Yan, X. (2012). CD146, an epithelialmesenchymal transition inducer, is associated with triplenegative breast cancer. Proceedings of the National Academy of Sciences of the United States of America.

Zhou, W., Fong, M.Y., Min, Y., Somlo, G., Liu, L., Palomares, M.R., Yu, Y., Chow, A., O'Connor, S.T.F., Chin, A.R., et al. (2014). Cancer-Secreted miR-105 destroys vascular endothelial barriers to promote metastasis. Cancer Cell 25, 501-515. 

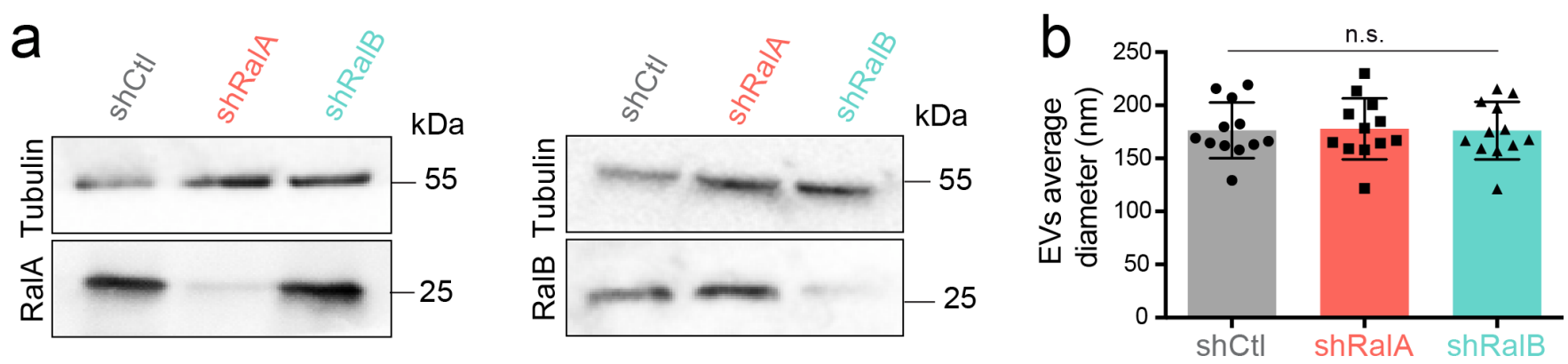

\section{Supplementary Figure 1}

Fig. 6. Supplementary Figure 1: a) Representative western blots showing tubulin, RalA (left) and RalB (right) expressions in 4T1 shControl, shRalA and shRalB cells. b) Graph showing the average diameter of the EVs isolated from $4 \mathrm{~T} 1 \mathrm{shControl,} \mathrm{shRalA}$ and shRalB cells measured by nanoparticle tracking analysis. Each dot represents one experiment (12 independent experiments; One Way Anova followed by Bonferroni's Multiple Comparison Test)

Supplementary material Table 1: EVs RNA analysis. Sheet a: RNAs overexpressed in EVs from 4T1 shCtl cells Vs EVs from shRalA cells Sheet b: RNAs overexpressed in EVs from 4T1 shRalA cells Vs EVs from shCtl cells Sheet c: RNAs overexpressed in EVs from 4T1 shCtl cells Vs EVs from shRalB cells Sheet d: RNAs overexpressed in EVs from 4T1 shRalB cells Vs EVs from shCtl cells

Table 2: EVs proteomic analysis. Sheet a: Proteins identified in EVs from 4T1 shCtl cells Sheet b: Proteins overexpressed in EVs from 4T1 shCtl cells Vs EVs from shRalA cells Sheet c: Proteins overexpressed in EVs from 4T1 shRalA cells Vs EVs from shCtl cells Sheet d: Proteins overexpressed in EVs from 4T1 shCtl cells Vs EVs from shRalB cells Sheet e: Proteins overexpressed in EVs from 4T1 shRalB cells Vs EVs from shCtl cells Sheet f: Proteins overexpressed in EVs from 4T1 shCtl cells Vs EVs from shRalA cells and EVs from shRalB cells 


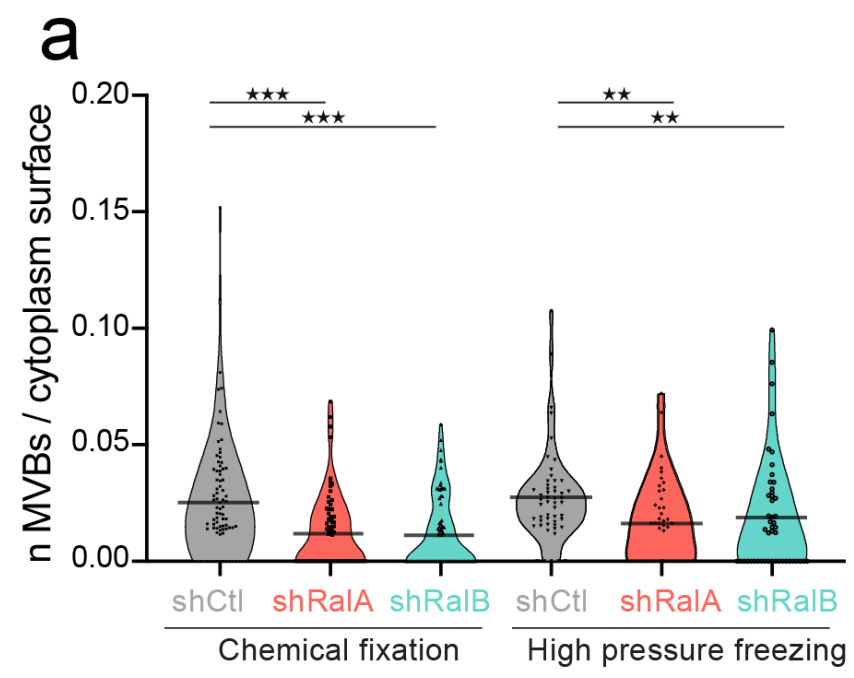

b
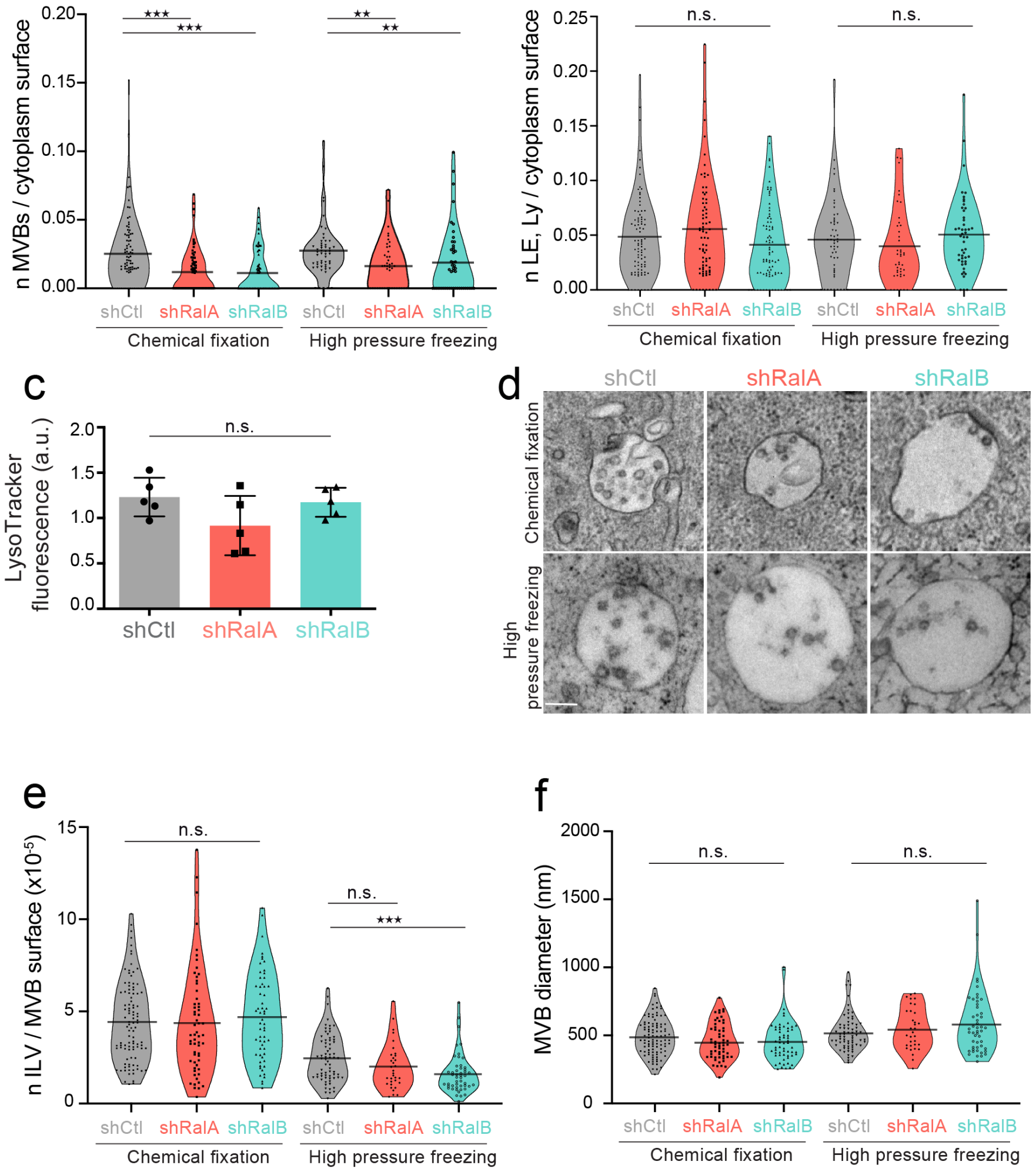

\section{Supplementary Figure 2}

Fig. 7. Supplementary Figure 2: Electron microscopy analysis of endosomes in the absence of RalA or RalB a-b) Graph showing the number of MVB (a) or endolysosomes (b) per cytoplasm surface in electron microscopy analysis performed by chemical fixation or high pressure freezing on 4T1 shControl, shRalA and shRalB cells. Each dot represents one field of view. Kruskal-Wallis test followed by Dunn's Multiple Comparison Test. c) FACS analysis of 4T1 shControl, shRalA and shRalB cells stained with Lysotracker. Each dot represents one experiment (5 independe $\neg$ t experiments; Kruskal-Wallis test followed by Dunn's Multiple Comparison Test). d) Representative electron micrographs of MVBs in 4T1 shControl, shRalA and shRalB cells after chemical fixation or high-pressure freezing. Scale bar: 200nm. e-f) Number of ILV per MVB surface (e)

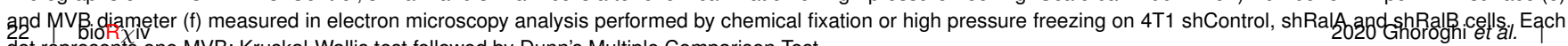
dot represents one MVB; Kruskal-Wallis test followed by Dunn's Multiple Comparison Test. 
bioRxiv preprint doi: https://doi.org/10.1101/2020.07.10.196691; this version posted July 10, 2020. The copyright holder for this preprint (which was not certified by peer review) is the author/funder. All rights reserved. No reuse allowed without permission.
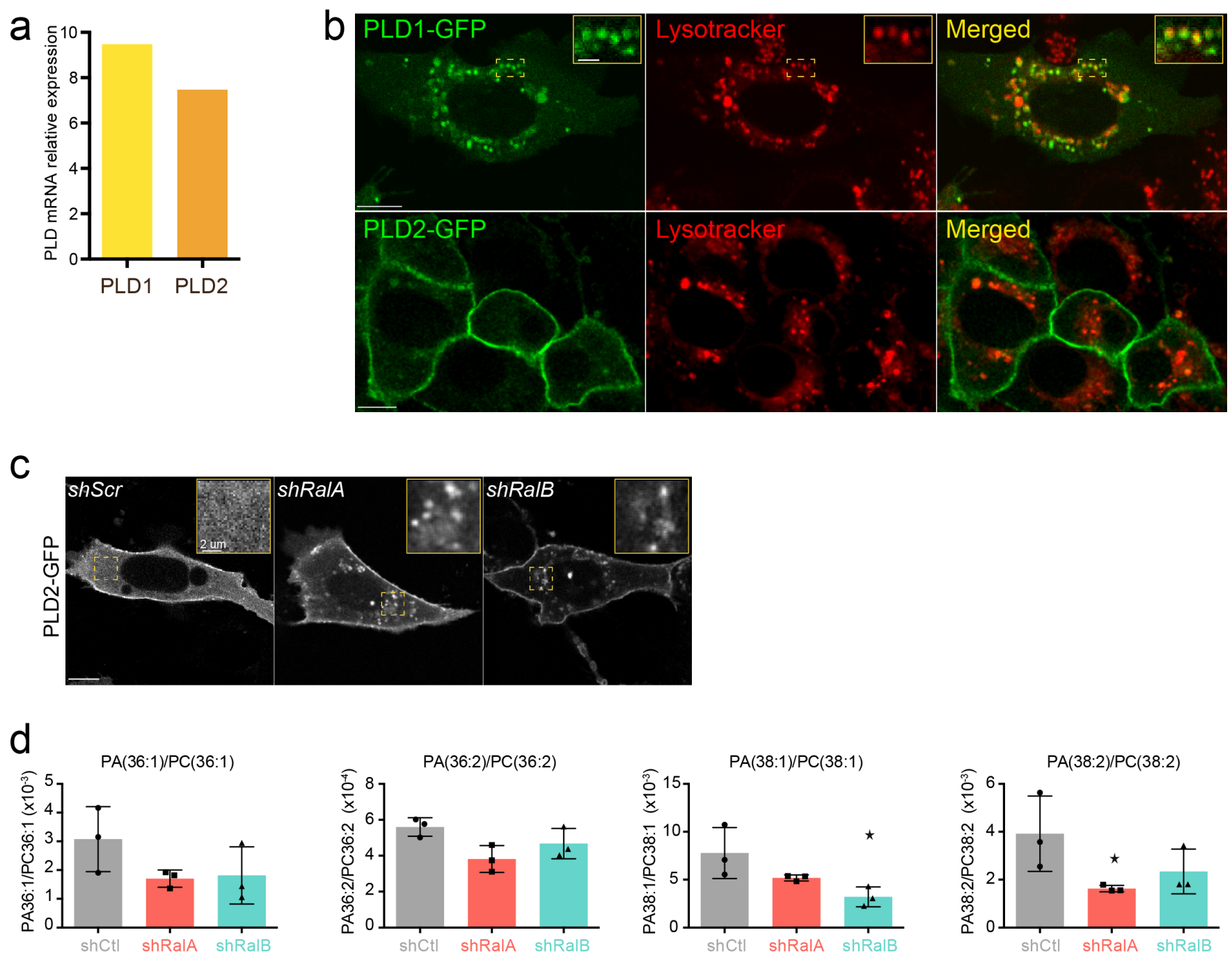

\section{Supplementary Figure 3}

Fig. 8. Supplementary Figure 3: PLD1 and PLD2 in 4T1 cells. a) PLD1 and PLD2 expression in 4T1 shControl cells quantified by qRT-PCR and normalized by gapdh expression. b) Representative confocal images showing PLD1-GFP and PLD2-GFP sub-cellular localization in 4T1 cells stained with lysotracker. Scale bars: $10 \mu \mathrm{m}$ c) Representative confocal images of PLD2-GFP localization in shControl, shRalA and shRalB cells. Scale bar: $10 \mu \mathrm{m}$ d) PA/PC ratio of species known to be targeted by PLD1 identified in EVs isolated from shControl, shRalA and shRalB cells. Each dot represents one experiment; 3 independent experiments. 
bioRxiv preprint doi: https://doi.org/10.1101/2020.07.10.196691; this version posted July 10, 2020. The copyright holder for this preprint (which was not certified by peer review) is the author/funder. All rights reserved. No reuse allowed without permission.

a
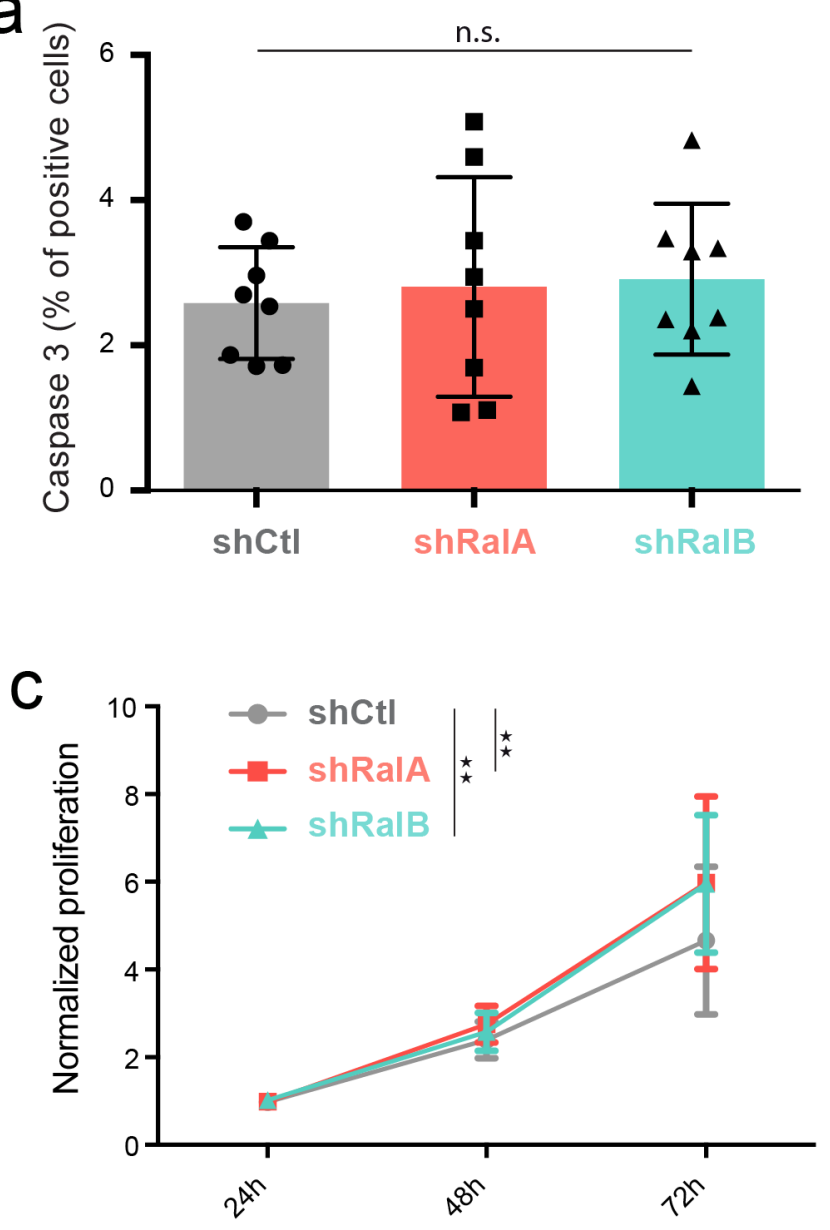

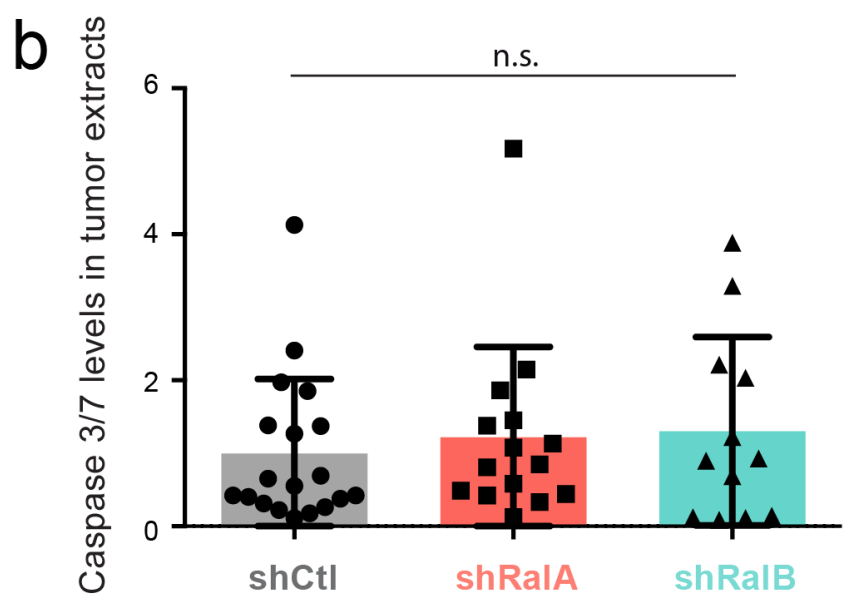

d

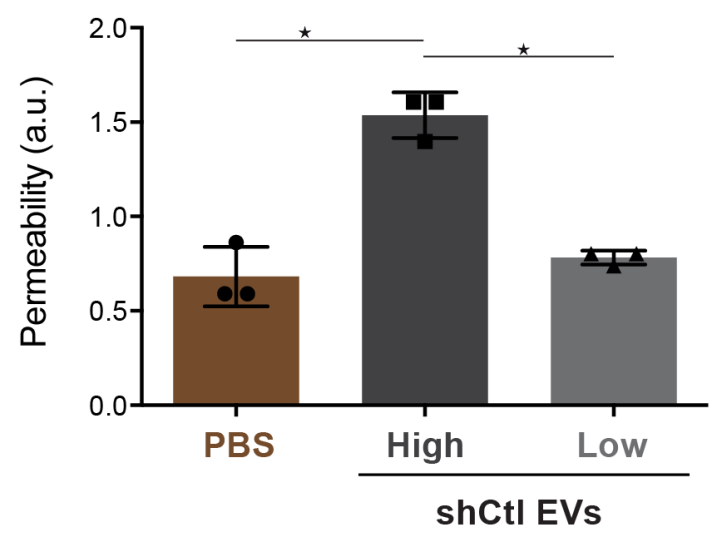

\section{Supplementary Figure 4}

Fig. 9. Supplementary Figure 4: a) Graph showing the percentage of cells expressing caspase 3 assessed by immunofluorescence on breast primary tumors. Each dot represents one mouse (8 mice taken from 2 independent experiments; one Way Anova followed by Bonferroni's Multiple Comparison Test). b) Graph showing the caspase3/7 expression levels on protein extracts from breast primary tumors. Each dot represents (12 to 19 mice from 2 independent experiments; Kruskal-Wallis test followed by Dunn's Multiple Comparison Test) c) Graph showing the proliferation of $4 \mathrm{~T} 1$ shControl, shRalA and shRalB cells over time in an in vitro assay. Two-way ANOVA followed by Bonferroni's Multiple Comparison Test ( 3 independent experiments with $n=5$ ). d) Graph showing the effect of high and low doses of $4 \mathrm{~T} 1$ EVs on permeabilisation of a HUVEC monolayer in vitro. The graph represents the normalized amount of fluorescent dextran that crossed the endothelial barrier. Each dot represents one experiment (3 independent experiments; One Way Anova permutation test followed with fdr multicomparison test). 


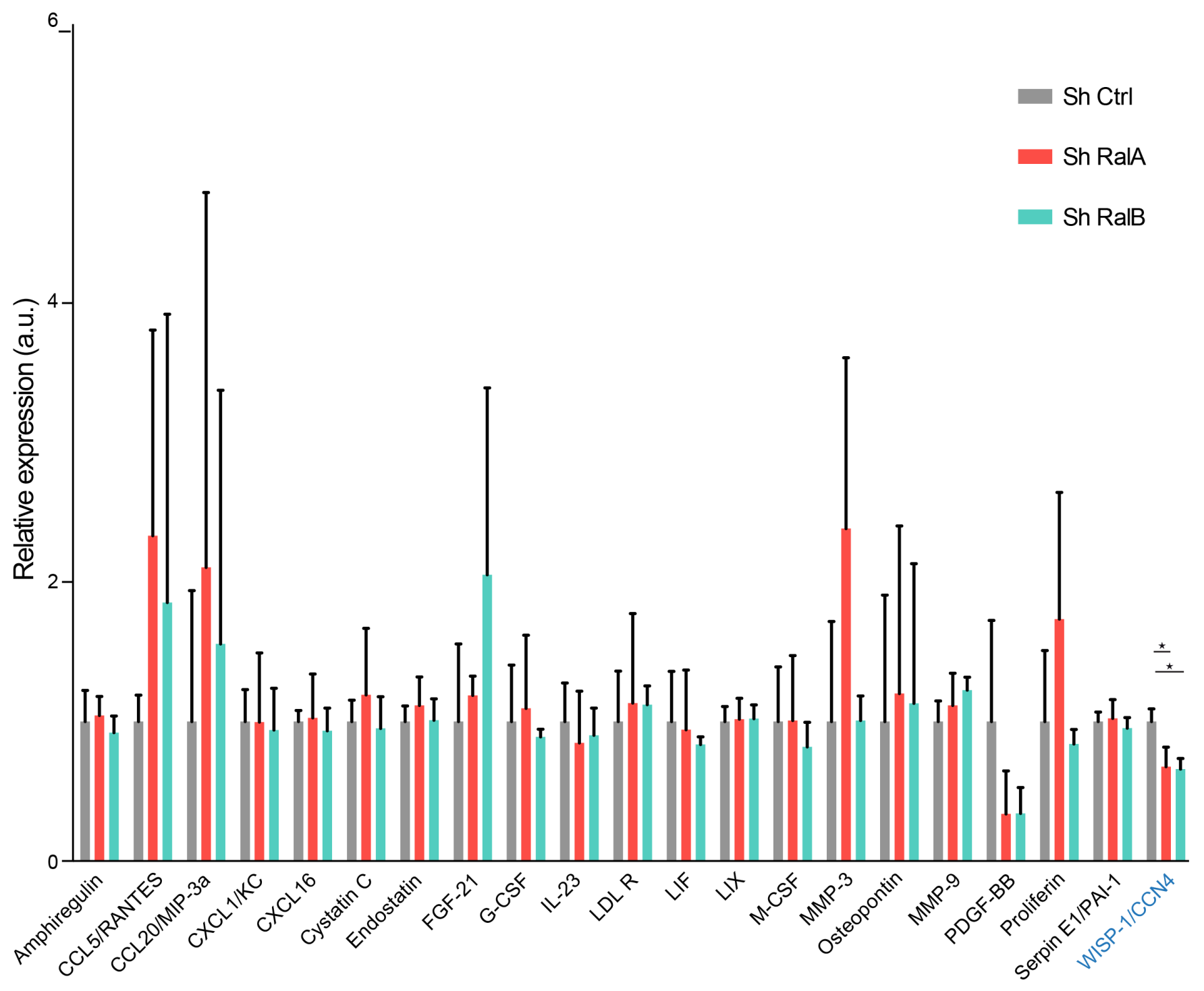

\section{Supplementary Figure 5}

Fig. 10. Supplementary Figure 5: Soluble secretome of $4 \mathrm{~T} 1$ shControl cells compared to $4 \mathrm{~T} 1$ shRalA or $4 \mathrm{~T} 1$ shRalB cells $(3$ independent experiments; One Way Anova permutation test followed with pairwise permutation test with fdr correction). 

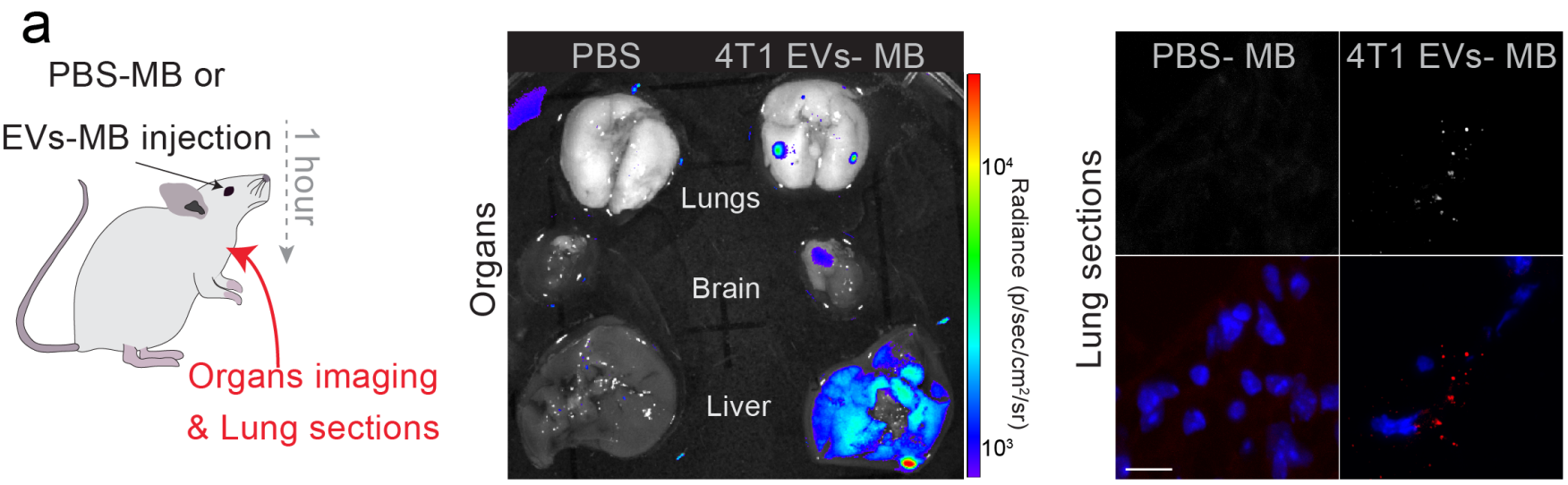

\section{b}
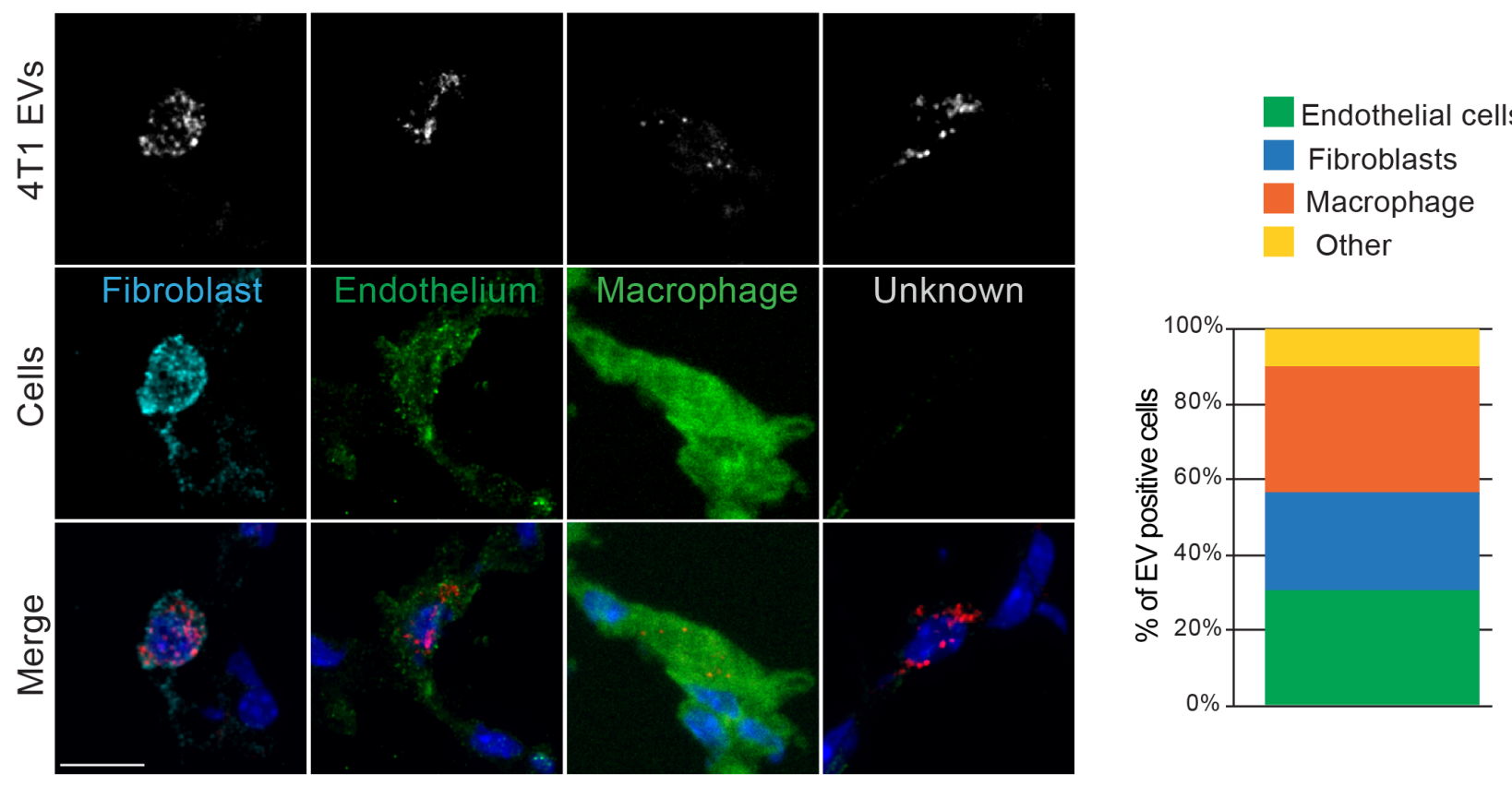

C

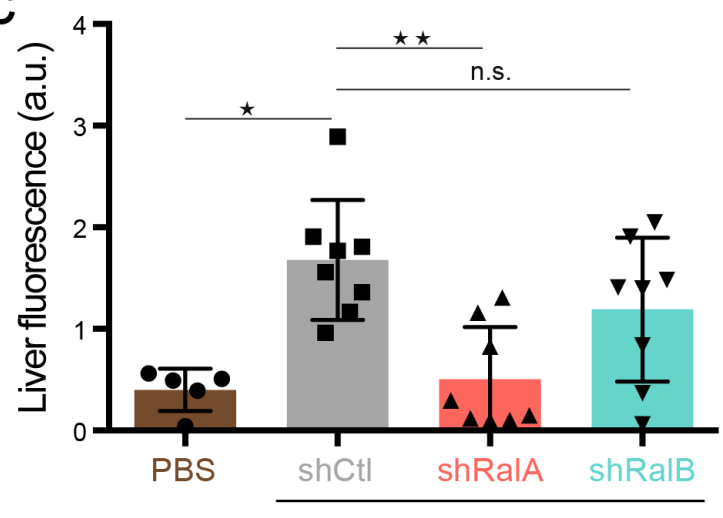

EVs

\section{Supplementary Figure 6}

Fig. 11. Supplementary Figure 6: $4 T 1$ EVs organotropism (a) Organs (left) and lungs sections (right) harvested from mice injected with PBS-MB or 4T1 MB-EVs 1h after intravenous injection. Scale bars: $10 \mu \mathrm{m}$. (b) Representative confocal images showing the identity of lung cells accumulating fluorescently labeled $4 \mathrm{~T} 1 \mathrm{EVs}$. Scale bars: 10 $\mu \mathrm{m}$. Graphs show the percentage of endothelial cells, fibroblasts, macrophages and cells of unknown identity among EV positive cells (108 positive cells from 6 mice taken from 2 independent experiments). c) Graph showing liver accumulation of fluorescent EVs isolated from shControl, shRalA and shRalB cells. Equal numbers of EVs were injected intravenously one hour before organ harvesting ( 8 mice taken from 2 independent experiments; Kruskal-Wallis test followed by Dunn's Multiple Comparison Test). 
bioRxiv preprint doi: https://doi.org/10.1101/2020.07.10.196691; this version posted July 10, 2020. The copyright holder for this preprint (which was not certified by peer review) is the author/funder. All rights reserved. No reuse allowed without permission.
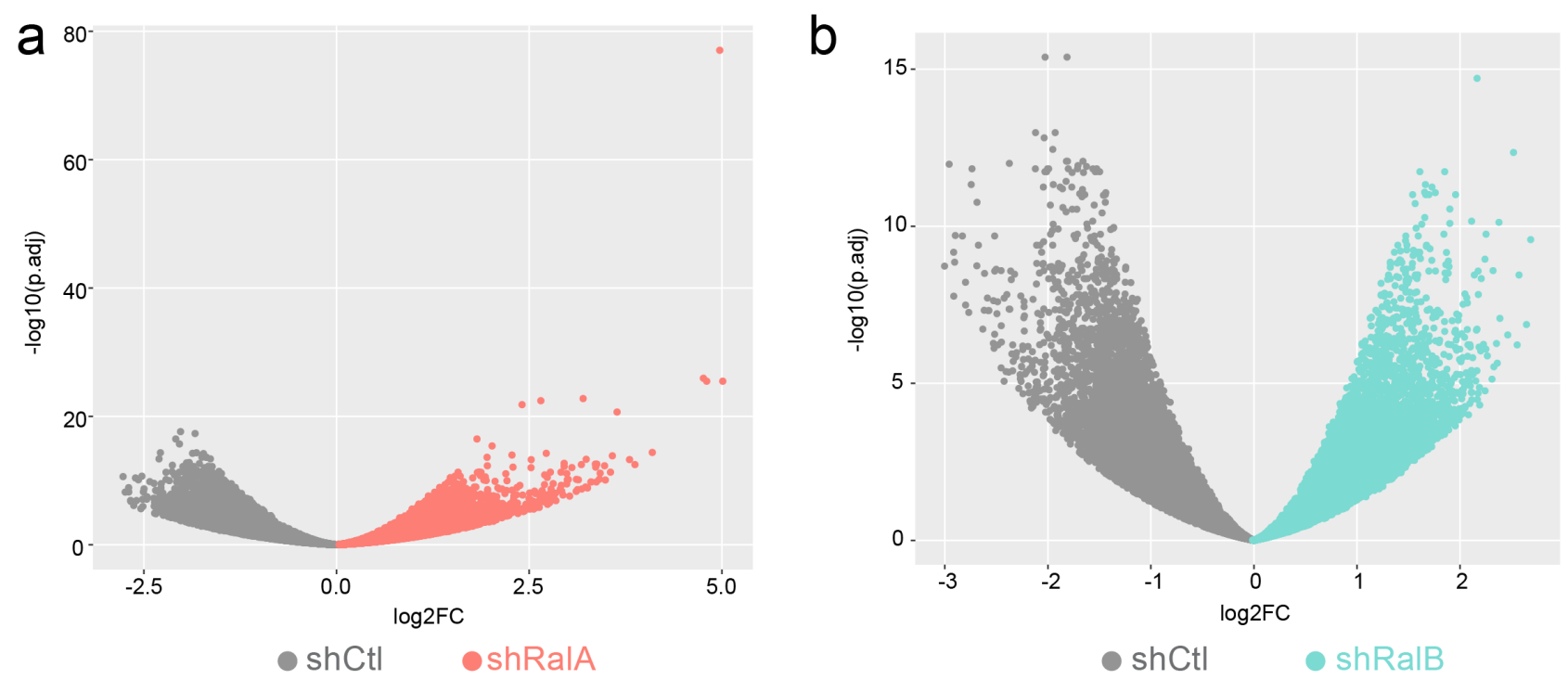

\section{C \\ GO terms}

Biological process

Molecular function

Cellular component

Voltage gated ion channel

Keratin filaments

Myosin filaments

mRNA enrichment analysis

Up in shRalA EVs
Cytoplasmic translation
Ribonucleoprotein assembly
Constituent of Ribosome
mRNA binding
Cytosolic ribosome
Lysosomal membrane

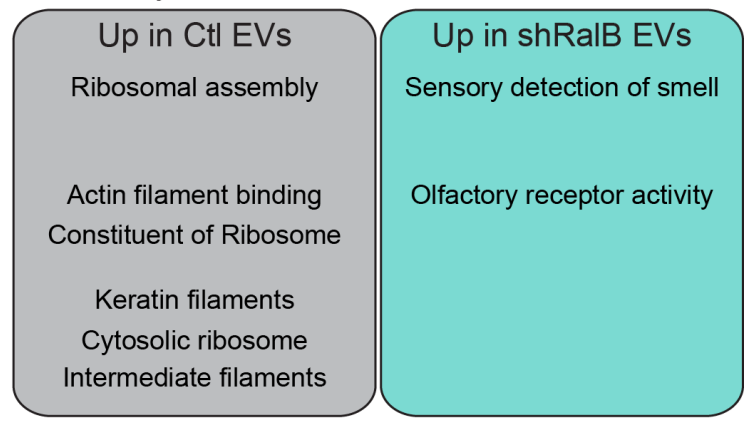

\section{Supplementary Figure 7}

Fig. 12. Supplementary Figure 7: RNA content of EVs from shControl, shRalA and shRalB cells a) Volcano plots comparing the mRNA present in EVs isolated from shControl and shRalA cells. b) Volcano plots comparing the mRNA present in EVs isolated from shControl and shRalB cells. c) GO term analysis of the mRNA present in EVs from shControl, shRalA and shRalB cells. 

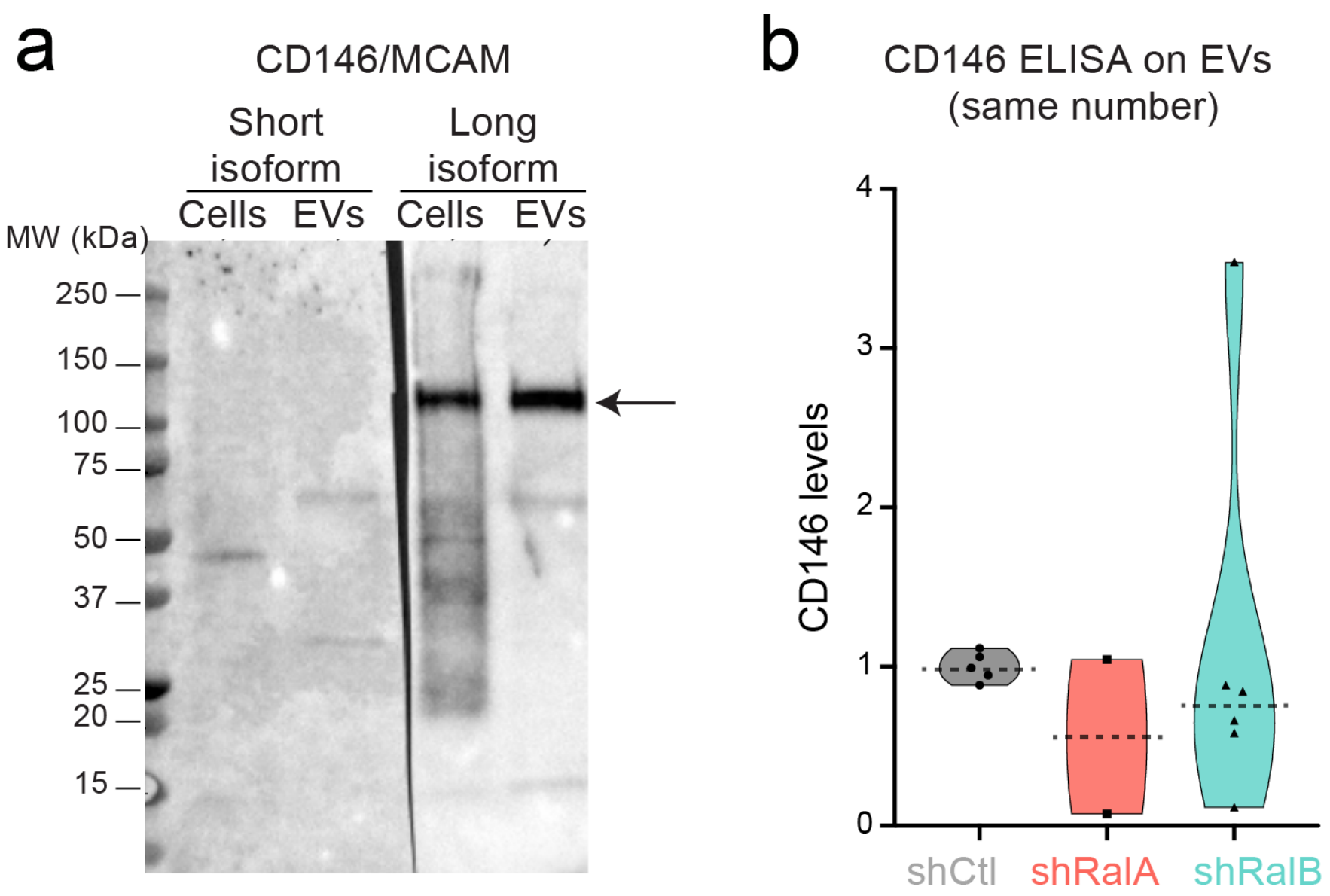

\section{Supplementary Figure 8}

Fig. 13. Supplementary Figure 8: $4 \mathrm{~T} 1$ cells and EVs express CD146/MCAM long isoform. a) Western blots on $4 \mathrm{~T} 1$ cell and EVs extracts using antibodies selectively recognizing CD146/MCAM short (left) and long (right) isoforms. b) Anti-CD146 Elisa on EVs isolated from shControl, shRalA and shRalB cells. Each dot represents one replicate. Horizontal line represents the median. 